
\title{
$\begin{array}{ll}\text { Research Square } & \begin{array}{l}\text { They should not be considered conclusive, used to inform clinical practice, } \\ \text { or referenced by the media as validated information. }\end{array}\end{array}$ \\ Cationic Nanostructures for the Treatment of Inflammatory Bone Loss
}

\author{
Hanyao Huang \\ West China Hospital of Stomatology https://orcid.org/0000-0002-6805-0157 \\ Weiyi Pan \\ Sichuan University \\ Yi-Fan Wang \\ Huazhong University of Science and Technology \\ Hyesung Kim \\ Kangwon National University \\ Dan Shao \\ Columbia University \\ Baoding Huang \\ Columbia University
}

Tzu-Chieh Ho

Columbia University

Yeh-Hsing Lao

Columbia University

Chai Hoon Quek

Columbia University

Jiayu Shi

The University of Michigan

\section{Bing Shi}

West China School of Somatology

Sheng-Min Zhang

Huazhong University of Science and Technology

\section{Lei Zhao}

Sichuan University

Kam W. Leong ( $\sim$ kam.leong@columbia.edu )

Columbia University https://orcid.org/0000-0002-8133-4955

\section{Article}

Keywords: cationic nanostructures, periodontitis, bone loss 
Posted Date: January 12th, 2021

DOl: https://doi.org/10.21203/rs.3.rs-139744/v1

License: (c) (i) This work is licensed under a Creative Commons Attribution 4.0 International License. Read Full License

Version of Record: A version of this preprint was published at Nature Communications on October 7th, 2022. See the published version at https://doi.org/10.1038/s41467-022-33492-6. 


\section{Abstract}

Periodontitis is a common type of inflammatory bone loss and is a risk factor for systemic diseases. The pathogenesis of periodontitis relies on inflammatory dysregulation, which represents a target for new therapeutic strategies to treat periodontitis. Here we demonstrate that cell-free DNA (cfDNA) is correlated with periodontitis in patient samples, and that cfDNA/TLR9 interactions participate in the immune response of periodontitis. We then tested the hypothesis that removing cfDNA would benefit periodontitis treatment. To create nucleic acid-binding nanoparticles (NABNs) specific for periodontitis, we coated bone-mimicking selenium-doped hydroxyapatite nanoparticles with cationic polyamidoamine dendrimers (PAMAM-G3), and compared the activities of these NABNs with those of soluble PAMAM-G3 polymers. Both NABNs and PAMAM-G3 inhibited periodontitis-related inflammation in vitro by scavenging cfDNA, and alleviated inflammatory bone loss in a mouse model of ligature-induced periodontitis. Both cfDNA scavengers also regulated the mononuclear phagocyte system in a periodontitis environment, promoting the M1 over the M2 macrophage phenotype. However, NABNs showed greater therapeutic effects than PAMAM-G3 in terms of scavenging and reducing inflammation and bone loss in vivo. Our findings demonstrate the importance of cfDNA in periodontitis and the potential for using cfDNA-scavenging and hydroxyapatite-based NABNs to ameliorate inflammation and bone loss in periodontitis.

\section{Introduction}

Periodontitis is a common chronic disorder that involves oral microbe-related inflammatory bone loss and local destruction of periodontal tissues, and is associated with systemic diseases such as heart and lung disease. ${ }^{1}$ In periodontitis, microbial inflammatory insults and abnormal host-microbiota interactions inappropriately and continuously activate the immune system, leading to inflammatory bone loss. ${ }^{2,3}$ This proinflammatory immune response is initiated by toll-like receptors (TLRs) and other pattern-recognition receptors that detect and respond to pathogen-, damage-, and microbe-associated molecular patterns (PAMPs, DAMPs, and MAMPs). ${ }^{4}$

One type of molecular pattern that contributes to periodontitis is cell-free DNA (cfDNA). ${ }^{5}$ DNA fragmentation in gingival crevicular fluid is correlated with periodontitis. ${ }^{6}$ cfDNA includes endogenous nuclear and mitochondrial DNA released by damaged host cells, and exogenous bacterial or viral DNA. 7,8 Bacterial DNA (bDNA) stimulates TLR99-11 and activates the TLR9-mediated immune response that is essential in the pathogenesis of periodontitis. ${ }^{12}$ bDNAs appear to link periodontitis and systemic diseases, as periodontal bDNA has been found in serum, cardiac tissue, synovial fluid, and in the intrauterine environment in these diseases. ${ }^{12-14}$ cfDNA is critical to chronic inflammation related to bone loss in periodontitis. ${ }^{15,16}$ We hypothesized that removing cfDNA with cationic nanoparticle scavengers would alleviate the development and progression of periodontitis.

Polyamidoamine dendrimers (PAMAM-G3) are soluble, cationic nucleic acid-binding polymers (NABPs) that prevent TLR activation and inhibit inflammation, and have demonstrated therapeutic efficacy in 
preclinical studies of acute liver failure, ${ }^{17}$ lupus, ${ }^{18}$ cancer metastasis, ${ }^{19}$ influenza infection, ${ }^{20}$ and sepsis. ${ }^{21}$ We have shown that insoluble cationic nucleic acid-binding nanoparticles (NABNs) exhibit greater therapeutic efficacy than soluble NABPs and lower toxicity in murine sepsis and rheumatoid arthritis models. ${ }^{21-23}$ Here we develop NABNs specifically for periodontitis by coating bone-mimicking selenium-doped hydroxyapatite nanoparticles (SeHANs) with PAMAM-G3, and compare the therapeutic activity of these NABNs with that of soluble PAMAM-G3 polymers in vitro and in a ligature-induced periodontitis murine model. We describe differences in the mechanisms by which these NABNs and NABPs inhibit inflammation and alleviate bone loss in periodontitis.

\section{Results}

\section{cfDNA levels in periodontitis patients and fabrication of nucleic acid-binding nanoparticles}

cfDNA levels in saliva and serum were significantly higher in periodontitis patients than in healthy volunteers (Fig. 1A and B). Based on this finding and on the participation of cfDNA/TLR9 interactions in the periodontitis immune response, we hypothesized that a cfDNA scavenging strategy could alleviate periodontitis (Fig. 1C, bottom). We chose SeHANs as the core of a cfDNA-binding nanoparticle for treating periodontitis due to the bone-mimicking properties of hydroxyapatite ${ }^{24,25}$ and the osteogenic and antioxidant properties of selenium, ${ }^{26-29}$ and coated the SeHANs with cationic PAMAM-G3 polymer to create particles with positive charge (G3@SeHANs) (Fig. 1C, top). G3@SeHANs were better dispersed than bare SeHANs based on scanning electron microscopy (SEM) and transmission electron microscopy (TEM) (Figs. 1C and S1). PAMAM-G3 coating of the SeHANs was confirmed by elemental mapping (Fig. S1), X-ray photoelectronic spectroscopy (XPS) (Fig. 1D-F), Fourier-transform infrared spectroscopy (FTIR; Fig. 1G), glass transition temperature (Fig. 1H), zeta potential (Fig. 1I), and Brunauer-Emmett-Teller (BET) surface area analysis (Fig. 1J). The size of the particles increased slightly following coating (Fig. 1K), and the phase did not change-the particles remained insoluble in aqueous medium (Fig. 1L). PAMAM-G3 grafting reduced the selenium release rate of the nanoparticles (Fig. S2A), indicating slower particle degradation. The lower selenium content of G3@SeHANs than of bare SeHANs (Fig. S2B) was likely due to selenium release during grafting. G3@SeHANs exhibited higher binding affinity to DNA than bare SeHANs (Fig. S3A), with no observed cytotoxicity (Fig. S3B).

\section{G3@SeHANs inhibit periodontitis-related inflammation by scavenging cfDNA in vitro}

We first examined whether G3@SeHANs inhibited TLR activation by various anionic agonists by using HEK-TLR3, -TLR4, -TLR8, and -TLR9 reporter cells and corresponding TLR agonists: poly(I:C) dsRNA, lipopolysaccharide (LPS), ORN06 ssRNA, and CpG DNA, respectively (Figs. S4 and S5). Both G3@SeHANs and bare SeHANs inhibited CpG-induced TLR9 activation in a dose-dependent manner, but G3@SeHANs caused greater inhibition than bare SeHANs; $10 \mu \mathrm{g} / \mathrm{mL}$ of G3@SeHANs caused a similar level of inhibition as $2 \mu \mathrm{g} / \mathrm{mL}$ of soluble PAMAM-G3 (Fig. S4A-B). The G3@SeHANs also inhibited poly(l:C)induced TLR3 activation, LPS-induced TLR4 activation, and ORN06-induced TLR8 activation to a greater extent than bare SeHANs (Fig. S5). In RAW 264.7 macrophages, addition of $10 \mu \mathrm{g} / \mathrm{mL}$ of G3@SeHANs 
alone (without any TLR agonist) had no effect on levels of the proinflammatory cytokines TNF- $a$ and IL-6 (Fig. S4C-D). Together, G3@SeHANs exhibited greater ability to scavenge anionic TLR agonists and inhibit TLR activation than bare SeHANs, indicating the importance of the cationic PAMAM-G3 coating.

We next examined whether G3@SeHANs prevented cellular inflammation induced by saliva and serum from periodontitis patients. Saliva and serum from periodontitis patients but not from healthy donors induced TLR9 activation in HEK-Blue TLR9 cells (Fig. 2A); this TLR9 activation was reduced by adding G3@SeHANs or PAMAM-G3. Saliva from periodontitis patients also caused an increase in TNF- $a$ and IL-6 levels (Fig. 2B), and these increases were mitigated by G3@SeHANs and PAMAM-G3. Serum from periodontitis patients had a smaller effect on TNF-a and IL-6 levels than saliva (Fig. 2B).

We then investigated whether cfDNA isolated from saliva could activate the TLR9-mediated periodontitis cellular immune response, what types of cfDNA were involved, and the ability of G3@SeHANs to counteract these cfDNAs. cfDNA isolated from periodontitis saliva induced TLR9 activation, and both G3@SeHANs and PAMAM-G3 reduced this cfDNA-induced TLR9 activation (Fig. 2C). Genomic DNA (gDNA) and mitochondrial DNA (mtDNA) are DAMPs that initiate periodontal inflammation. mtDNA but not gDNA activated TLR9 (Fig. 2C) and significantly increased TNF- $\alpha$ and IL-6 levels in human and mouse macrophages (Figs. S6 and S7); these cytokine increases were inhibited by G3@SeHANs and PAMAM-G3. We then used two common types of gingival cells-human gingival keratinocytes (HGKs) and human gingival fibroblasts (HGFs) -to collect DAMPs. DAMPs from both cells and mitochondria (mtDAMPs) caused significant increases in proinflammatory cytokine release (Fig. S7) and TLR9 activation (Fig. S8), and these increases were mitigated by adding G3@SeHANs and PAMAM-G3. PAMPs such as CpG DNA also initiate periodontitis. CpG DNA induced TLR9 activation and increased TNF- $\alpha$ and IL- 6 levels (Figs. $2 \mathrm{C}$ and S7); these effects were inhibited by G3@SeHANs and PAMAM-G3. We then used two common periodontitis-related bacteria, Porphyromonas gingivalis and Fusobacterium nucleatum, to collect MAMPs. MAMPs also caused a significant increase in TLR9 activation, which was reduced by adding G3@SeHANs and PAMAM-G3 (Fig. S8). Together, both G3@SeHANs and PAMAM-G3 were able to inhibit periodontitis-related TLR9-mediated cellular inflammation induced by different types of cfDNA.

\section{G3@SeHANs reduce inflammatory bone loss in ligature-induced periodontitis in vivo}

We used a ligature-induced periodontitis mouse model described previously. ${ }^{30,31}$ In this model, significant bone loss was observed at approximately two weeks after placing the ligature. The dose of G3@SeHANs injected into the inflammatory site, $1 \mathrm{mg} / \mathrm{mL}$, was determined in a pilot study (Fig. S9). The ligature was placed in a circle around the second molar to obtain significant bone loss and related phenotypes. The four treatment groups were (1) no ligature (healthy control); (2) ligature, phosphate-buffered saline (PBS) (periodontitis control) PAMAM-G3; and (4) ligature, G3@SeHANs. PBS, PAMAM-G3, or G3@SeHANs were injected on both sides of mice into six sites around the ligature on days $0,3,6,9$, and 12 ; samples were collected on day 15 . Treatments were also smeared onto the gum around the ligature daily except on injection days (Fig. 3A-B). 
cfDNA and TNF-a levels in saliva and serum were significantly higher in periodontitis model mice than in normal mice; these proinflammatory cytokine levels were inhibited by treatment with G3@SeHANs and PAMAM-G3 (Fig. 3C-F). The cfDNA level in saliva increased in periodontitis model mice starting on day 12 (Fig. 3G). Treatment with G3@SeHANs but not PAMAM-G3 reduced the cfDNA level nearly to that of normal mice throughout the experiment. TNF-a levels in saliva had already increased by day 3; treatment with G3@SeHANs but not PAMAM-G3 significantly reduced TNF-a levels by day 15 (Fig. 3H). Together, cfDNA and TNF-a levels in both local and systemic environments (saliva and serum) were reduced by G3@SeHANs to a greater extent than by soluble PAMAM-G3. Both G3@SeHANs and PAMAM-G3 inhibited ligature-induced bone loss as observed by micro-computed tomography (micro-CT) imaging (Fig. 3I-J).

The ligatures caused a strong inflammatory response and epithelial destruction, observed by hematoxylin and eosin (H\&E) staining (Figs. 4A and S10A). Bone loss in the G3@SeHAN and PAMAM-G3 groups was less than in the untreated periodontitis group (Figs. 4A and S10A). Osteoclastic and osteogenic activity, indicated by enzyme activity staining with tartrate-resistant acid phosphatase (TRAP) and alkaline phosphatase (ALP), respectively, were higher in the untreated periodontitis group than in normal mice. The number of TRAP ${ }^{+}$cells was lower in the G3@SeHAN and PAMAM-G3 groups than in the untreated periodontitis group (Fig. 4B and Fig. S10B), indicating reduced osteoclastic activity. TLR9 expression was significantly higher in the untreated periodontitis group than in normal mice; both G3@SeHAN and PAMAM-G3 reduced this periodontitis-associated TLR9 expression (Fig. 4C-D). Treatment with G3@SeHANs and PAMAM-G3 also reduced TNF- $a$ and IL-6 levels, as well as mRNA levels of the NF-KB pathway transcription factors Rela, Tnf, and $/ / 6$ (Fig. 4E-I), indicating inhibition of NF-KB signaling.

To confirm the role of cfDNA in the pathogenesis of periodontitis and the function of the NABNs, we established another periodontitis model with an additional local injection of CpG DNA (Fig. S11A-B). Micro-CT imaging showed that bone loss in this CpG DNA group was slightly greater than in the original untreated periodontitis group; however, the differences were not significant (Fig. S11C-D). Cationic scavengers restrained the increased levels of both G3@SeHANs and PAMAM-G3 mitigated the increases in cfDNA and proinflammatory cytokine levels in the $\mathrm{CpG}$ DNA group versus the normal mice group, but G3@SeHANs had a larger effect (Fig. S11E). The epithelium was more severely damaged in the untreated CpG DNA group than in the G3@SeHAN- and PAMAM-G3-treated CpG DNA groups (Fig. S11F). The number of osteoclasts was highest in the untreated $\mathrm{CpG}$ group, and was reduced by treatment with G3@SeHANs and PAMAM-G3 (Fig. S11G). These results and the results above showed that G3@SeHANs and PAMAM-G3 alleviated cfDNA-induced inflammatory bone loss in the ligature-induced periodontitis model, and G3@SeHANs had a greater effect than PAMAM-G3.

The diversity of bacterial communities in the oral cavity of ligature-induced periodontitis mice was unaffected by administration of the cationic materials (Fig. S12A-D). In a principal component analysis (PCA) plot, samples from the untreated, PAMAM-G3, and G3@SeHAN groups clustered together, suggesting similar microbial community structures (Fig. S12E). Between-group differences were similar to within-group differences (Fig. S12F and Table S1), and the microbial compositions in the three groups were similar (Fig. S12G). In terms of safety, H\&E staining revealed no organ damage following local 
treatment with G3@SeHAN or PAMAM-G3 (Fig. S13). Ligature-induced periodontitis and scavenger treatment did not significantly alter the serum levels of safety-related biochemical parameters (Fig. S14AF).

\section{Scavenging mechanisms of G3@SeHANs and PAMAM-G3}

The results above indicated differences between G3@SeHANs and PAMAM-G3 in their in vitro scavenging and in vivo bone protection effects. Due to the large difference in size of the two cationic materials, we hypothesized that differences in activity were related to the difference in size and whether the two materials operated intracellularly or extracellularly. To examine the localization of the materials, we conducted an intracellular trafficking study in RAW 264.7 cells with Cy5.5-labeled CpG DNA (Cy5.5-CpG) and FITC-labeled PAMAM-G3 (FITC-G3) or FITC-labeled G3@SeHANs. When no cationic materials were added, $\mathrm{CpG}$ was found mainly in lysosomes at $4 \mathrm{~h}$ and $12 \mathrm{~h}$. FITC-G3 intensity was observed mainly in lysosomes and in the cytoplasm at these time points (Fig. S15 and Fig. 5A). In contrast, FITC-

G3@SeHANs were observed mainly extracellularly, and the amount of Cy5.5-CpG in lysosomes was lower at $4 \mathrm{~h}$ than when using FITC-G3. At $12 \mathrm{~h}$, most Cy5.5-CpG was colocalized with FITC-G3@SeHANs, and no Cy5.5 intensity was observed in lysosomes. This difference in nucleic acid scavenging location between G3@SeHANs (extracellular) and soluble PAMAM-G3 (intracellular) may help explain the observed differences in their therapeutic effects.

To examine intracellular nucleic acid-sensing signal transduction, TLR9 and downstream NF-KB pathway activation were analyzed by qRT-PCR. Saliva from periodontitis patients, mtDNA, and CpG DNA were administered as stimuli to murine macrophage RAW264.7 cells. G3@SeHAN treatment consistently reduced the expression of TLR9 and NF-KB pathway mRNA relative to the untreated group, whereas PAMAM-G3 had no consistent effect (Fig. S16). Together, these results suggest that the two cationic materials inhibit cfDNA-induced inflammation via different mechanisms, involving scavenging cfDNA in different locations, as summarized in Fig. 5B.

\section{G3@SeHANs regulate the mononuclear phagocyte system}

T-cell-mediated host immunity is the dominant immune response in the pathogenesis of periodontitis, and dendritic cells (DCs) are the primary antigen presenting cells that activate T-cells. ${ }^{32}$ In the in vivo study, the numbers of T cells and DCs were elevated in the untreated periodontitis group versus the normal mice group, and treatment with the two cfDNA scavengers mitigated the increase in T cells and DCs (Figs. S17 and S18). Macrophage polarization in gingival tissue is related to the pathogenesis of periodontitis. ${ }^{33,34} \mathrm{M} 1$ macrophages are proinflammatory while M2 macrophages promote repair. M1 macrophages were found in the untreated periodontitis group but not in the normal mice group, and scavenger treatment of periodontitis mice reduced the number of M1 macrophages (Fig. S19). In contrast, scavenger treatment increased the number of M2 macrophages. We further examined the differentiation of human monocyte THP-1 cells to confirm the relationship between scavenger treatment and macrophage polarization. THP-1 cells were pretreated with phorbol myristate acetate (PMA) to 
induce differentiation from monocytes to M0-type macrophages. ${ }^{35}$ The morphology and surface markers of cells were altered by treatment with saliva from periodontitis patients (Figs. S20 and S21). The percentage of $\mathrm{CD} 197^{+}$cells (M1 marker) was higher in response to periodontitis saliva than to healthy saliva, whereas the percentage of $\mathrm{CD}^{2} 6^{+}$cells (M2 marker) was lower (Fig. 6A-C and Fig. S22). These results indicated that periodontitis saliva was more prone to promote inflammation than healthy saliva. The percentages of $\mathrm{CD} 83^{+}$and $\mathrm{CD} 86^{+}$cells (DC markers) were significantly higher in response to periodontitis saliva than healthy saliva (Fig. S23). Together, macrophage polarization and dendrite development occurred in response to treatment with saliva from periodontitis patients.

We then tested whether G3@SeHANs and PAMAM-G3 regulated this macrophage polarization and the functions of these macrophage subsets. For the M1 phenotype, levels of TNF- $a$ and IL- 6 increased following exposure to periodontitis saliva, but this increase was significantly reduced by treatment with G3@SeHANs and PAMAM-G3 (Fig. S24). For the M2 phenotype, exposure to periodontitis saliva increased IL-10 and reduced BMP-2 secretion, but had no effect on the TGF- $\beta$ level; treatment with G3@SeHANs and PAMAM-G3 further increased the IL-10 level and also increased TGF- $\beta$ and BMP-2 levels.

Periodontitis saliva significantly reduced the percentages of $\mathrm{CD} 14^{+}, \mathrm{CD} 68^{+}$, and $\mathrm{CD} 36^{+}$cells versus healthy saliva, indicating induction of differentiation of monocytes and M0-type macrophages to inflammatory status and suppression of differentiation to M2-type macrophages. Addition of the cationic scavengers had no significant effect on the proportion of M1 marker-positive cells, but profoundly inhibited the reduction in $\mathrm{CD}_{14}{ }^{+}, \mathrm{CD} 68^{+}$, and $\mathrm{CD} 36^{+}$cells (Fig. 6D-E). These results demonstrated that G3@SeHANs and PAMAM-G3 inhibited the proinflammatory polarization of macrophages. Thus, nucleic acid scavengers can regulate the mononuclear phagocyte system in a periodontitis environment.

\section{Discussion}

The correlation between local inflammatory bone loss in different locations and systemic diseases has been an active area of research for several decades. ${ }^{36}$ The link between periodontitis and systemic disease is related to the presence of microbes, but specific biomarkers for periodontitis remain unclear. Recent evidence suggests that dysregulation of nucleic acid-sensing pathway activation triggers imbalances in the innate immune system, leading to chronic periodontitis. ${ }^{1}$ cfDNAs of different origins are critical in many inflammatory diseases, and elevated cfDNA is found in the serum of patients with systemic diseases such as rheumatoid arthritis, sepsis, atherosclerosis, and cancer. ${ }^{37-40}$ As systemic diseases are correlated with periodontitis, we hypothesized that cfDNA may also be elevated in periodontitis, and that targeting the interaction between cfDNA and nucleic acid-sensing pathway activation may be useful for periodontitis treatment and prevention of periodontitis-related systemic diseases. We found that levels of circulating and local cfDNA were elevated in periodontitis mice and patients. Changes in cfDNA levels in saliva during periodontitis establishment in mice were similar to those previously observed in synovial fluid during establishment of rheumatoid arthritis in mice. ${ }^{23}$ Although the underlying mechanisms-and most interestingly, whether accumulation of circulating cfDNA 
corresponds to local inflammation-have yet to be fully elucidated, scavenging cfDNA to block abnormal cfDNA-sensing pathways appears useful for treating uncontrolled localized inflammatory bone loss in periodontitis.

We fabricated a periodontitis-specific nucleic-acid binding nanoparticle to scavenge cfDNA. PAMAM-G3 is a widely studied nucleic acid-binding polymer that can neutralize proinflammatory TLR9 agonists, but its toxicity hinders its clinical translation. Therefore, we applied PAMAM-G3 as the functional component of a larger, insoluble nucleic-acid binding nanoparticle. The resulting G3@SeHANs were less toxic than soluble PAMAM-G3 and possess a selenium-doped hydroxyapatite nanoparticle (SeHAN) core that is favorable for clinical translation. Selenium is an essential nutrient that maintains the proper physiological functions of multiple organ systems. ${ }^{41}$ Blood selenium has been found to be inversely correlated with the rate of bone turnover. ${ }^{42}$ Mechanistic studies have suggested that the biological functions of selenium mainly involve antioxidant defense. ${ }^{26,27}$ Our previous work has shown that SeHANs have great potential for treating bone cancers and promoting osteogenic differentiation. ${ }^{28,29}$ SeHANs set a high standard for the future development and evaluation of multifunctional biomaterials for dental disease therapy.

We next determined whether the NABNs could inhibit periodontitis-related inflammation by targeting local cfDNA. We found that saliva and serum from periodontitis patients enhanced TLR9 activation, but that activation by saliva was much stronger than by serum, which might be explained by the constant exposure of saliva to microbes in periodontitis. During the innate immune response, MAMPs continuously accumulate because of dying bacteria. ${ }^{43}$ Local inflammation also leads to cell death and increases the level of DAMPs. ${ }^{44}$ These phenomena explain why TRL9 activation by saliva was greater than by serum. Both NABNs and soluble PAMAM-G3 inhibited inflammation triggered by saliva and serum. The next question was whether cfDNA was the main functional component that caused inflammation, and whether the NABNs alleviated this inflammation. cfDNA from saliva, mtDNA, and CpG DNA all activated the TLR9 pathway. CpG motifs in DNA are correlated with TLR9 activation and inflammatory bone loss. ${ }^{38}$ CpG motif-rich DNA sequences have been found in synovial fluid and serum from rheumatoid arthritis patients. ${ }^{38}$ In periodontitis, CpG-rich bDNA and mtDNA may be why saliva cfDNA induces TLR9 activation. ${ }^{45,46}$ The application of the NABNs reduced inflammatory activation by depleting these cfDNAs in vitro.

Blocking the interaction of cfDNA and cfDNA-sensing pathways appears to be a potential therapeutic approach to treat periodontitis. One interesting finding in this study was that a localized cfDNA blockade influenced the circulating cfDNA level. We carried out two in vivo studies to demonstrate this effect. First, we used NABNs to treat ligature-induced periodontitis to test the therapeutic effect of the cfDNA-blocking strategy. With local injection of NABNs into periodontal tissues, the cfDNA level significantly decreased not only in saliva, but also in serum. Due to the association between cfDNA and systemic diseases, and between periodontitis and systemic diseases, eliminating cfDNA may be useful therapeutic approach to treat periodontitis and periodontitis-related systemic diseases. With the reduced level of cfDNA, alveolar bone loss was inhibited and inflammatory responses were dampened. DNA-sensing pathways play 
essential roles in periodontitis pathogenesis, and depleting cfDNA should affect these pathways. TLR9, the most widely-investigated pathway in DNA-sensing and cfDNA-scavenging studies, is also important in periodontitis. ${ }^{1}$ TLR9 activation in gingival epithelium was significantly greater than that in normal epithelium, and was reduced by the cationic scavengers. CpG DNA injections were performed to provide further evidence of the function of cfDNA and scavengers. A slight increase in alveolar bone loss in the CpG DNA-enhanced untreated group was found. We noticed that the distance of the cemento-enamel junction to the alveolar bone crest (CEJ-ABC) and bone resorption areas of the untreated group in the second in vivo study (with CpG DNA injection) were not the same as in the first study, possibly due to a difference between different batches of mice. Nevertheless, the NABNs consistently inhibited periodontitis and reduced levels of local and circulating proinflammatory factors.

In previous studies, we showed that release of selenium from SeHANs was mediated by degradation, and that selenium was an antibacterial factor. ${ }^{24,29}$ Here we wanted to avoid disrupting the balance of microbiota in a study related to oral or gut research because serious complications may occur when this balance is disrupted. 16S RNA analysis showed that NABNs did not influence the oral microbiota community, a favorable result in view of the theory that a smaller influence on the microbiota is indicative of a better medical intervention. ${ }^{47}$ The lack of disruption of microbiota could be due to the injection of NABNs into tissues; any selenium released from the tissue may not influence the balance of oral surface microbiota. We did not include the normal mice group (without ligature) in the 16S RNA analysis because the ligature around the tooth would certainly change the microbiota in the oral cavity of mice.

Although both NABNs and PAMAM-G3 reduced inflammation, differences between the effects of these cationic scavengers were evident. PAMAM-G3 is soluble and is internalized by cells, whereas the large G3@SeHANs remain extracellular. A strength of our study is that we confirmed differences in therapeutic activity between a soluble, internalized scavenger and an insoluble, extracellular scavenger. Based on qRT-PCR analysis of DNA-sensing signaling, the larger, insoluble scavenger appeared to achieve more stable depletion of danger signals. Another important aspect that requires further study is what happened to cfDNA absorbed by the NABPs. Soluble polymers scavenge cfDNA intracellularly and can also be used to deliver cfDNA into cells in gene delivery. Intracellular scavenged cfDNA could further activate the proinflammatory pathway. Extracellular scavengers might avoid this possibility because the cfDNA is scavenged before cell entry, preventing interactions between agonists and TLR receptors.

Macrophages, DCs, and other cells in the mononuclear phagocyte system play an important role in periodontitis. ${ }^{33,48,49}$ Clinical observations have shown that M1/M2 macrophages and DCs are correlated with the stage of periodontitis, and that saliva affects the periodontitis environment. Our in vivo results are consistent with these clinical findings. A previous study of a scavenger strategy to treat sepsis showed that cfDNA promoted the proinflammatory phenotype of macrophages; ${ }^{21}$ we obtained similar results here. Periodontitis saliva can also affect the phenotype of DCs. We showed that periodontitis saliva contained more inflammatory stimulatory components than normal saliva. However, unlike the in vivo results of the sepsis study, ${ }^{21}$ here, NABPs and PAMAM-G3 did not reduce the M1 phenotype but did 
promote the M2 phenotype. This difference in the effect of cationic scavengers on macrophage polarization could be related to the different ways that scavengers were administered. Our results suggest that macrophage polarization can be regulated by cationic scavengers. Further, we showed that local cfDNA scavenging and mitigation of inflammation can influence circulating cfDNA levels, suggesting that local factors can enter the circulation and influence systemic health. Although we did not determine the function of cfDNA in systemic diseases, we propose cfDNA as a new periodontitis-systemic disease link.

In summary, this study revealed cfDNA as an important biomarker in periodontitis and as a new target for periodontitis treatment. The nucleic acid-binding nanoparticle approach shows excellent potential for alleviating periodontitis. To harness the proven therapeutic activity of soluble PAMAM-G3 polymer while reducing its toxicity, we developed nucleic acid-binding nanoparticles consisting of bone-mimicking selenium-doped hydroxyapatite coated with PAMAM-G3 that favorably alleviated inflammation by scavenging cfDNA, resulting in inhibition of cfDNA-mediated inflammatory alveolar bone loss. Together, our findings suggest a new nanoparticulate scavenger approach for treating periodontitis and shed light on the development of safe, effective, and clinically translatable cfDNA nanostructures for periodontitis.

\section{Materials And Methods}

\section{Materials and reagents}

Calcium nitrate tetrahydrate, trisodium phosphate, sodium selenite, linoleic acid, octadecylamine, anhydrous ethanol, nitrate acid, PAMAM-G3, anti-TLR9 rabbit polyclonal antibody, fluorescein isothiocyanate isomer I (FITC), 4',6-diamidine-2'-phenylindole dihydrochloride (DAPI), brain-heart infusion broth (BHI), agar, hemin and menadione, cell counting kit-8 (CCK-8), and PMA were purchased from Sigma-Aldrich (St. Louis, MO, U.S.A.). Poly(I:C) (tlrl-picw), ultrapure LPS (t|rl-peklps), ORN06/LyoVec (tlrlorn6), ODN BW006 (tlrl-bw006), ODN 2395, and QUANTI-Blue medium were purchased from InvivoGen (San Diego, CA, U.S.A). A bicinchoninic acid (BCA) protein assay kit, H\&E staining kit, defibrinated sheep blood, and Schaedler broth were purchased from Solarbio (Beijing, China). Paraformaldehyde (4\%) was purchased from Servicebio (Wuhan, Hubei, China). A TRAP/ALP staining kit was purchased from FUJIFILM Wako Chemicals (Osaka, Japan). Anti-iNOS (D6B6S) rabbit monoclonal antibody (cat. \#13120), anti-arginase-1 (D4E3M) XP rabbit monoclonal antibody (cat. \#93668), and PathScan Sandwich ELISA Lysis Buffer were purchased from Cell Signaling Technology (Danvers, Massachusetts, U.S.A). Alexa Fluor 594-conjugated goat anti-rabbit lgG (H+L) (cat. \#ZF-0516) was purchased from ZSGBBIO (Beijing, China). Anti-CD11c (N418) Armenian hamster monoclonal antibody (cat. \#14-0114-82), antiF4/80 (BM8) rat monoclonal antibody (cat. \#14-4801-82), human anti-CD80 antibody (cat. \#46-0809-41), human anti-CD83 antibody (cat. \#17-0839-4), human anti-CD86 antibody (cat. \#12-0869-41), human antiCD209 antibody (cat. \#25-2099-41), human anti-CD14 antibody (cat. \#12-0149-41), human anti-CD68 antibody (cat. \#25-0689-41), and human anti-CD197 antibody (cat. \#17-1979-41) were purchased from eBioscience (San Diego, CA, U.S.A). An anti-rabbit HRP-DAB IHC Detection Kit (cat. \#CTS005) was purchased from Novus (Minneapolis, MN, U.S.A). Human anti-CD14 antibody (cat. \#325603) was 
purchased from BioLegend (San Diego, California, U.S.A). Human anti-CD36 antibody (cat. \#555454) was purchased from BD Biosciences. Alexa Fluor 647-conjugated goat anti-Armenian hamster IgG antibody (cat. \#ab173004) and anti-CD3 rabbit polyclonal antibody (cat. \#ab5690) were purchased from Abcam (Cambridge, UK). Dylight 488-conjugated AffiniPure goat anti-rat IgG $(\mathrm{H}+\mathrm{L})$ (cat. \#E032240) was purchased from EARTHOX (San Francisco, CA, U.S.A). ProLong Gold Antifade Mountant (P36930), UltraPure salmon sperm DNA, a Quant-iT PicoGreen dsDNA assay kit, LysoTracker Red DND-99, a Mitochondria Isolation Kit, ELISA kits for human and mouse TNF-a, human and mouse IL-6, human IL-10, human TGF- $\beta$, and BMP-2, a TaqMan Advanced miRNA cDNA Synthesis Kit, and a RevertAid First Strand cDNA Synthesis Kit were purchased from Thermo Scientific (Waltham, Massachusetts, U.S.A). An iScript One-Step RT-PCR Kit with SYBR Green was purchased from Bio-Rad (Hercules, CA, U.S.A.). A 2'3'-cGAMP ELISA kit was purchased from Cayman Chemical (Ann Arbor, MI, U.S.A.). Dulbecco's modified Eagle's medium (DMEM), RPMI-1640 medium, fetal bovine serum (FBS), and 0.25\% trypsin-EDTA were purchased from Gibco (Carlsbad, CA, U.S.A.). A dermal cell basal medium and keratinocyte growth kit were purchased from the American Type Culture Collection (ATCC, Manassas, VA, U.S.A.). An RNApure Total RNA Fast Extraction Kit (cat. \#RP1202) was purchased from Bioteke Corporation (Wuxi, Jiangsu, China). Cy5.5-labeled CpG 1826 and primers were synthesized and purchased from Integrated DNA Technologies (IDT, Coralville, IA, U.S.A.). All other reagents were commercially available and were used as received (Table S2).

\section{Synthesis of SeHANs}

SeHANs were synthesized using a modified liquid-solid-solution (LSS) method. Briefly, $1.18 \mathrm{~g}$ calcium nitrate tetrahydrate was dissolved in $25 \mathrm{~mL}$ deionized water and then mixed with an organic solution composed of $1.5 \mathrm{~g}$ octadecylamine, $12 \mathrm{~mL}$ linoleic acid, and $48 \mathrm{~mL}$ anhydrous ethanol. Thereafter, $461.25 \mathrm{mg}$ trisodium phosphate and $48.65 \mathrm{mg}$ sodium selenite were dissolved in $20 \mathrm{~mL}$ deionized water and added dropwise to the above mixture. After stirring at room temperature for $10 \mathrm{~min}$, the suspension was transferred into a hydrothermal reactor and allowed to react for $12 \mathrm{~h}$ at $110{ }^{\circ} \mathrm{C}$. The resulting precipitates were washed with anhydrous ethanol and deionized water at least six times and collected by centrifugation, and were stored in anhydrous ethanol at $4{ }^{\circ} \mathrm{C}$.

\section{Synthesis of G3@SeHANs}

To decorate SeHANs with PAMAM-G3 (G3), SeHANs (1 mg) were mixed with G3 (10 mg) in PBS (pH 7.4, $0.2 \mathrm{~mL}$ ), and the mixture was incubated for $12 \mathrm{~h}$ at room temperature with shaking. G3-coated SeHANs (G3@SeHANs) were centrifuged at 3000 rpm for 5 min to remove unbound G3 and were washed three times with PBS. The size and zeta potential of G3@SeHANs were measured with a Zetasizer (Nano ZS90, Malvern Panalytical).

\section{Characterization of SeHANs and G3@SeHANs}

X-ray diffraction (XRD, PANalytical B.V., Holland) and FTIR (Vertex 70, Bruker, Germany) analyses were performed to investigate the phase composition and functional structure of SeHANs and G3@SeHANs. 
Morphology studies were performed using field emission scanning electron microscopy (FSEM, FEl, Holland) and field emission transmission electron microscopy (FTEM, FEl, Holland). Elemental mapping was performed using FTEM. The valence state of selenium in nanoparticles and PAMAM-G3 grafting on the surface of SeHANs were determined by XPS (Kratos, Japan). PAMAM-G3 content was detected by thermogravimetric analysis (TGA, PerkinElmer, U.S.A.) in an $\mathrm{N}_{2}$ atmosphere. Specific surface area was measured by BET analysis (Micromeritics, U.S.A.).

\section{Selenium content in SeHANs and G3@SeHANs}

The amount of selenium in synthesized nanoparticles was quantified using an inductively coupled plasma optical emission spectrometer (ICP-OES, Prodigy Plus, Leeman Labs, U.S.A.). Nanoparticles (2.5 $\mathrm{mg}$ ) were dissolved in $1 \mathrm{~mL}$ of $70 \mathrm{wt} \%$ nitric acid and diluted to $10 \mathrm{~mL}$ with $1 \mathrm{wt} \%$ nitric acid before ICPOES analysis.

\section{Patient sample collection}

Saliva and serum samples from 11 patients with periodontitis and 14 healthy volunteers were obtained from West China Hospital of Stomatology, Sichuan University. Sample collection was performed with the approval of the Ethics Committee of West China Hospital of Stomatology, Sichuan University.

Periodontitis was confirmed by periodontal pockets that showed bleeding upon probing (with $\geq 5.0 \mathrm{~mm}$ probing depth), $\geq 3.0 \mathrm{~mm}$ of clinical attachment loss, and radiographic evidence of alveolar bone loss on at least two teeth per quadrant. ${ }^{50}$ Periodontal tissue from healthy volunteers showed no redness, no bleeding on probing, and no clinical attachment loss, and radiographs showed periodontal areas without bone loss.

\section{Biodegradation and selenium release by SeHANs and G3@SeHANs}

Selenium release by SeHANs and G3@SeHANs, which represented their biodegradation, was detected by ICP-OES in three different solutions: PBS pH 7.4, saliva from healthy donors, and saliva from periodontitis patients. Saliva was collected and sterilized with a $0.22-\mu \mathrm{m}$ filter (Millipore), then nanoparticles were prepared as a suspension at $2.5 \mathrm{mg} / \mathrm{mL}$ with these three solutions in $1.5-\mathrm{mL}$ tubes. Thereafter, the tubes were placed in a water bath shaker at $37^{\circ} \mathrm{C}$. At each time point, the tubes were centrifuged at $12,000 \mathrm{rpm}$ for $15 \mathrm{~min}$ to collect $1 \mathrm{~mL}$ of supernatant, and $1 \mathrm{~mL}$ of fresh buffer solution was added to restore the volume. The collected supernatant was digested with $1 \mathrm{~mL}$ of $70 \mathrm{wt} \%$ nitric acid and diluted to $10 \mathrm{~mL}$ with $1 \mathrm{wt} \%$ nitric acid before ICP-OES analysis.

\section{DNA-binding efficiency of SeHANs and G3@SeHANs}

Bare SeHANs or G3@SeHANs (200 mg) were mixed with salmon sperm DNA (2-30 mg in 1 mL Tris-EDTA buffer) and incubated for $3 \mathrm{~h}$ at room temperature with shaking. The mixture was centrifuged at 3000 rpm for $5 \mathrm{~min}$, and the supernatant containing unbound DNA was collected and analyzed with a Quant-iT PicoGreen dsDNA assay to determine the amount of DNA bound to the nanoparticles. 


\section{Bacterial culture}

Porphyromonas gingivalis and Fusobacterium nucleatum strains were provided by the State Key Laboratory of Oral Diseases, the West China School of Stomatology, Sichuan University. Porphyromonas gingivalis and were stored in glycerol broth at $-80^{\circ} \mathrm{C}$. The bacteria were cultured in brain heart infusion $(\mathrm{BHI})$ or $5 \%$ defibrinated sheep blood agar, both supplemented with $5 \mathrm{mg} / \mathrm{mL}$ hemin-menadione. Fusobacterium nucleatum was cultured in Schaedler broth. Bacteria were incubated in an anaerobic environment $\left(90 \% \mathrm{~N}, 5 \% \mathrm{CO}, 5 \% \mathrm{H}_{2}\right)$ at $37^{\circ} \mathrm{C}$.

\section{DAMP and MAMP isolation}

HGF-1 and primary gingival keratinocytes (PGKs) (ATCC) were maintained according to ATCC protocols and were harvested for DAMP collection. Mitochondria were collected from HGFs with a mitochondrion isolation kit and were used for DAMP isolation. Bacteria (Porphyromonas gingivalis and Fusobacterium nucleatum) were used for MAMP isolation. Cells and bacteria were collected in 1.5-mL tubes and ultrasonicated for $10 \mathrm{~min}$. The concentration of DNA was measured. For DNA measurements, mixtures were centrifuged at 10,000 rpm for $5 \mathrm{~min}$, and supernatants were collected for DNA quantification. Isolated DAMPs and MAMPs were stored at $-80^{\circ} \mathrm{C}$ until further use.

\section{In vitro cytotoxicity assay}

RAW 264.7 cells were seeded into 96 -well culture plates at a density of $2 \times 10^{4}$ cells/well and cultured until the cells were fully attached. Then the cells were treated with different concentrations of NABNs or soluble PAMAM-G3 for $24 \mathrm{~h}$ and were counted with a cell counting kit-8 (CCK-8).

\section{Extraction and quantification of cfDNA}

Extraction of cfDNA from saliva or serum was performed with a DNeasy Blood \& Tissue Kit (QIAGEN, Germany). Concentrations of cfDNA in saliva, serum, DAMPs, and MAMPs were measured with a QuantiT PicoGreen double-stranded DNA Assay Kit.

\section{In vitro TLR3, TLR4, TLR8, and TLR9 activation assay}

Stable hTLR3-, 4-, 8-, and 9-overexpressing HEK-Blue cells were purchased from InvivoGen (San Diego, CA, U.S.A.) and were initially propagated in DMEM with $10 \%(\mathrm{v} / \mathrm{v}) \mathrm{FBS}$ and maintained in growth medium supplemented with selective antibiotics. Before treatment, certain numbers of HEK-Blue hTLR cells $\left(5 \times 10^{4}\right.$ cells/well hTLR3, $2.5 \times 10^{4}$ cells/well hTLR4, $4 \times 10^{4}$ cells/well hTLR8, and $8 \times 10^{4}$ cells/well hTLR9 cells) were seeded and cultured in basal DMEM overnight in 96-well plates, then stimulated with the appropriate agonists $(1 \mu \mathrm{g} / \mathrm{mL}$ low molecular weight poly(l:C) for hTLR3, $10 \mathrm{ng} / \mathrm{mL}$ ultrapure LPS for hTLR4, 0.5 $\mu \mathrm{g} / \mathrm{mL}$ ORN06/LyoVec for hTLR8, and $1 \mu \mathrm{g} / \mathrm{mL}$ ODN BW006 for hTLR9). In the scavenger-treated groups, PAMAM-G3 $(2 \mu \mathrm{g} / \mathrm{mL})$, SeHANs $(10 \mu \mathrm{g} / \mathrm{mL})$, or G3@SeHANs $(10 \mu \mathrm{g} / \mathrm{mL})$ were added $30 \mathrm{~min}$ prior to adding the agonist. After $24 \mathrm{~h}$, the activation of reporter cells was determined with QUANTI-Blue medium. One microliter of human saliva, $5 \mu \mathrm{L}$ of human serum, DAMPs from PGKs (cfDNA concentration: 1 
$\mu \mathrm{g} / \mathrm{mL}$ ), DAMPs from HGFs (cfDNA concentration: $1 \mu \mathrm{g} / \mathrm{mL}$ ), DAMPs from mitochondria (cfDNA concentration: $500 \mathrm{ng} / \mathrm{mL}$ ), MAMPs from Porphyromonas gingivalis (cfDNA concentration: $1 \mu \mathrm{g} / \mathrm{mL}$ ), and MAMPs from and Fusobacterium nucleatum (cfDNA concentration: $1 \mu \mathrm{g} / \mathrm{mL}$ ) were also prepared as agonists to test the function of cationic scavengers. Briefly, $50 \mu \mathrm{L}$ supernatant from each well of the cell culture plate was transferred to $150 \mu \mathrm{L}$ QUANTI-blue medium to test the corresponding embryonic alkaline phosphatase (SEAP) activity, which was first loaded into the empty well and incubated at $37^{\circ} \mathrm{C}$ for the reaction, and OD620 was measured.

\section{In vitro anti-inflammatory assays}

RAW 264.7 cells were seeded and cultured in basal DMEM overnight at $2 \times 10^{4}$ cells per well in a 96 -well plate. PAMAM-G3 $(2 \mu \mathrm{g} / \mathrm{mL})$ or G3@SeHANs $(10 \mu \mathrm{g} / \mathrm{mL})$ were added in a final volume of $200 \mu \mathrm{L} 30 \mathrm{~min}$ prior to addition of agonist. ODN BW006 $(1 \mu \mathrm{g} / \mathrm{mL}), 1 \mu \mathrm{L}$ of human saliva, $5 \mu \mathrm{L}$ of human serum, DAMPs from PGKs (cfDNA concentration: $1 \mu \mathrm{g} / \mathrm{mL}$ ), DAMPs from HGFs (cfDNA concentration: $1 \mu \mathrm{g} / \mathrm{mL}$ ), DAMPs from mitochondria (cfDNA concentration: $500 \mathrm{ng} / \mathrm{mL}$ ), MAMPs from Porphyromonas gingivalis (cfDNA concentration: $1 \mu \mathrm{g} / \mathrm{mL}$ ), and MAMPs from Fusobacterium nucleatum (cfDNA concentration: $1 \mu \mathrm{g} / \mathrm{mL}$ ) were then added into the well. After incubation for $24 \mathrm{~h}$, the supernatants were collected and TNF-a and IL-6 levels were measured using ELISA kits.

THP-1 cells were purchased from ATCC and cultured in RPMI-1640 media supplemented with 10\% FBS and selective antibiotics. Briefly, $8 \times 10^{4}$ cells were plated in 96 -well plates in $200 \mu \mathrm{L} \mathrm{RPMI}$ media plus 25 $\mathrm{ng} / \mathrm{mL}$ PMA for $48 \mathrm{~h}$ of treatment to induce differentiation to macrophages. For polarization, THP-1 macrophages were treated with PMA for $48 \mathrm{~h}$, and periodontitis saliva was added during the final $18 \mathrm{~h}$ of treatment. PAMAM-G3 $(2 \mu \mathrm{g} / \mathrm{mL})$ or G3@SeHANs $(10 \mu \mathrm{g} / \mathrm{mL})$ were added in a final volume of $200 \mu \mathrm{L} 30$ min prior to adding saliva. After incubation, supernatants were collected and TNF- $a$, IL-6, TGF- $\beta$, IL-10, and BMP-2 levels were measured with ELISA kits.

\section{Animal model establishment and treatment}

The design of the animal experiment was approved by the Ethics Committee of West China School of Stomatology (Chengdu, China). Male BALB/C mice (7-8 weeks old) were purchased from Dossy Experimental Animals Co., Ltd. (Chengdu, Sichuan, China) and maintained at the Experimental Animal Center of West China Second University Hospital (Chengdu, Sichuan, China). Animals were fed standard food and water ad libitum, and light was provided according to natural circadian rhythms.

The murine experimental model of periodontitis was established by placing a ligature (Coated VICRYL Suture, 5-0; Ethicon I J\&J Medical Devices, Somerville, New Jersey, U.S.A.) around the cervix of the maxillary second molar. ${ }^{51}$ General anesthesia was administered by the intramuscular injection of Zoletil50 (50 mg/kg; Virbac, Carros, Grasse, France). The local administration of the materials (PAMAMG3: $200 \mu \mathrm{g} / \mathrm{mL}$; G3@SeHANs: $1 \mathrm{mg} / \mathrm{mL}$ ) or PBS (in the control group) was accomplished by microinjection using a microsyringe $(25-\mu \mathrm{L}$ syringe with a 32-gauge needle; Hamilton Company, Reno, Nevada, U.S.A.) into gingival tissue at six sites ( $5 \mu \mathrm{L} /$ site) around the ligature, including the mesiobuccal 
gingiva, distobuccal gingiva, mesiopalatal gingiva, distopalatal gingiva, and mesial and distal gingival papilla. To reduce the amount of anesthesia and local damage to the gingival tissue, microinjections were performed every three days (on days $0,3,6,9$, and 12 ); on experimental days without injection, the materials and PBS were noninvasively smeared on the gingiva using disposable microapplicators (M6500-SF purple; TPC Advanced Technology, Inc., City of Industry, California, U.S.A.).

To further determine the effect of pathological DNA on periodontitis, local injection of CpG (ODN2395, $100 \mu \mathrm{g} / \mathrm{mL}$; InvivoGen, San Diego, California, U.S.A.) was performed in addition to ligature placement. The injection of $\mathrm{CpG}$ was performed as described above $30 \mathrm{~min}$ after each injection of materials or PBS.

\section{Animal body fluid and tissue sample collection}

Saliva samples were collected before each injection after ligature placement and before sacrificing the animal, on days $3,6,9,12$, and 15 . The saliva secreted by mice within 3 min after anesthesia was pipetted and collected. After centrifugation at 15,000 rpm for $10 \mathrm{~min}$, the supernatant was collected and stored at $-80^{\circ} \mathrm{C}$ until further testing.

Animals were sacrificed 15 days after ligature placement. Oral microbiome samples were collected using an ultrafine polystyrene swab (25-800 1PD 50; Puritan Medical Products, Guilford, Maine, U.S.A.) according to a published protocol, ${ }^{52}$ and were stored at $-80{ }^{\circ} \mathrm{C}$ until further testing. Blood samples were collected by eyeball extirpation under general anesthesia, coagulated at room temperature for $30 \mathrm{~min}$, then centrifuged at $3,000 \mathrm{rpm}$ for $10 \mathrm{~min}$ to collect serum. Serum was stored at $-80^{\circ} \mathrm{C}$ until further testing.

After blood collection, animals were sacrificed by cervical dislocation. First, half of the maxilla on a random side was dissected and rinsed in cold PBS. The crown of the molars, maxillary bone, and buccal soft tissues were removed from samples, and only the gingival tissues and alveolar bone around the three molars were collected. The trimmed maxillary sample was temporarily placed on dry ice and stored at $-80^{\circ} \mathrm{C}$ for further testing. Then, the other side of the maxilla, heart, liver, spleen, lung, and kidney were dissected and fixed in $4 \%$ paraformaldehyde at $4{ }^{\circ} \mathrm{C}$ overnight. The fixed samples were rinsed with tap water for $6 \mathrm{~h}$ and were stored in $70 \%$ ethanol at $4{ }^{\circ} \mathrm{C}$ until further testing.

\section{Micro-CT reconstruction and bone resorption quantitative analyses}

Fixed maxillary samples were used first for micro-CT scanning (vivaCT80; SCANCO Medical, Brüttisellen, Switzerland). Scanning was performed at $145 \mathrm{~mA}$ and $55 \mathrm{kVp}$ every $10 \mu \mathrm{m}$ at high resolution. Further measurements of the vertical distance between the cementoenamel junction (CEJ) and alveolar bone crest $(A B C)$ and three-dimensional reconstruction were performed with Mimics Research v19.0.0 (Materialise; Leuven, Belgium). The mesial and distal CEJ-ABC $(\mu \mathrm{m})$ of the maxillary second molar were recorded as the mean of the numbers measured on five mesiodistal sectional planes. After threedimensional reconstruction, a standard figure of the buccal and palatal aspect of the maxillary molars was obtained, on which the bone resorption area $\left(\mathrm{mm}^{2}\right)$ was measured using Photoshop CC (Adobe; San 
Jose, California, USA). The bone resorption area was defined as the area enclosed by the continuous line of $\mathrm{CEJ}$ of the three molars and $\mathrm{ABC}$.

\section{Histological analysis}

After micro-CT scanning, maxillary samples were decalcified in $10 \%$ ethylene diamine tetra acetic acidPBS solution at $4{ }^{\circ} \mathrm{C}$ for three weeks. Then, the decalcified maxilla and fixed organs were dehydrated, embedded in paraffin wax, and sectioned $(4 \mu \mathrm{m})$. H\&E staining and TRAP/ALP staining were performed using a staining kit.

For IF analysis, DCs were detected by an anti-CD11c Armenian hamster monoclonal antibody and Alexa Fluor 647-conjugated goat anti-Armenian hamster IgG antibody. Macrophages were detected by an antiF4/80 rat monoclonal antibody and DyLight 488-conjugated AffiniPure goat anti-rat IgG ( $\mathrm{H}+\mathrm{L})$. In addition, markers of macrophage subpopulations were co-stained to identify the macrophage composition. M1-type and M2-type macrophages were stained by an anti-iNOS and anti-arginase-1 rabbit monoclonal antibody, respectively. 4',6-diamidino-2-phenylindole (DAPI) was used to stain the cell nucleus. All IF-stained slices were mounted using ProLong Gold Antifade Mountant. Images were captured using an Upright Automated Fluorescence Microscope (BX63; Olympus, Tokyo, Japan), and relative quantitative analyses were performed by comparing the integral optical density (IOD) of the positive area using Image-Pro Plus v6.0.0 (Media Cybernetics; Rockville, Maryland, U.S.A.).

For immunohistochemistry (IHC) analysis, the expression and location of TLR9 was detected by an antiTLR9 rabbit polyclonal antibody. T cells were detected by an anti-CD3 rabbit polyclonal antibody. IHC was performed using an Anti-Rabbit HRP-DAB IHC Detection Kit. Images were captured using an Aperio ScanScope slide scanner (Leica Biosystems, Wetzlar, Germany), and relative quantitative analyses were performed as described above.

\section{Protein and RNA extraction from animal samples}

Maxillary samples that were stored at $-80^{\circ} \mathrm{C}$ were homogenized using a high-speed tissue grinder (KZ-Il; Servicebio, Wuhan, Hubei, China). Total RNA was extracted by using an RNApure Total RNA Fast Extraction Kit, and the quality and concentration of total RNA was assessed by a Nanodrop 2000 (Thermo Scientific, Waltham, Massachusetts, U.S.A.). Then, reverse transcription was performed using a RevertAid First Strand cDNA Synthesis Kit, and complementary DNA was stored at $-20^{\circ} \mathrm{C}$ until further testing.

Total protein used for ELISA was extracted using PathScan Sandwich ELISA Lysis Buffer. The concentration of total protein was measured by the BCA method. The total protein sample was stored at $-80^{\circ} \mathrm{C}$ until further testing.

\section{Cytokine concentration analysis}


TNF- $\alpha$ and IL-6 levels in culture supernatants of RAW 264.7 cells and concentrations of TNF- $\alpha$, IL-6, TGF- $\beta$, IL-10, and BMP-2 in culture supernatants of THP-1 cells were determined using ELISA kits.

\section{Quantitative real-time polymerase chain reaction assay}

TRIzol reagent was used to extract total RNA, and $1 \mu \mathrm{g}$ of total RNA was reverse-transcribed using an iScript One-Step RT-PCR Kit with SYBR Green. Quantitative polymerase chain reaction (PCR) was then performed using SYBR Green. Amplified transcripts were quantified using the comparative $C t$ method.

\section{Biochemical parameter analysis}

Alanine transaminase (ALT), aspartate transaminase (AST), blood urea nitrogen (BUN), total bilirubin (TBIL), phosphocreatine kinase (CK), and creatinine (CRE) were measured on a chemistry analyzer (Chemray-800, Rayto) with reagents and settings recommended by the manufacturer.

\section{Fluorescent labeling of cationic scavengers and the intracellular uptake of G3@SeHANs}

FITC (150 mg) dissolved in DMSO (50 mL) was slowly added to PAMAM-G3 (3 mg) dispersed in sodium bicarbonate buffer $(50 \mathrm{mM}, \mathrm{pH}$ 9.0, $0.4 \mathrm{~mL}$ ) with vigorous stirring. The mixture was incubated for $12 \mathrm{~h}$ at $4{ }^{\circ} \mathrm{C}$. FITC-labeled PAMAM-G3 (FITC-G3) was purified using an Amicon Ultra0.5 mL 10K filter (Merck Millipore, Germany). FITC-G3 was used to coat SeHANs for further confocal laser scanning microscopy (CLSM) imaging.

RAW 264.7 cells were seeded onto a cover glass at a density of 10,000 cells $/ \mathrm{cm}^{2}$. After $12 \mathrm{~h}, \mathrm{Cy} 5.5$ labeled CpG (1 mg/mL) was added to cells with FITC-G3 $(2 \mathrm{mg} / \mathrm{mL})$ or FITC-G3@SeHANs $(10 \mathrm{mg} / \mathrm{mL})$ and incubated for $12 \mathrm{~h}$. After $4 \mathrm{~h}, 8 \mathrm{~h}$, and $12 \mathrm{~h}$ of treatment, the cells were washed with PBS, stained with LysoTracker Red DND-99 and DAPI, and mounted for CLSM.

\section{Flow cytometry analysis}

THP-1 cells were treated with the method described in the in vitro anti-inflammatory assay section. Then, the cells were collected for flow cytometry analysis. Cells were washed with fluorescence-activated cell sorting (FACS) buffer (1x PBS plus 0.5\% FBS) and blocked with Human TruStain FcX (Fc Receptor Blocking Solution). To identify DCs and monocytes, cells were stained with antibodies against human CD14, CD80, CD83, CD86 and CD209 for $30 \mathrm{~min}$ at $4{ }^{\circ} \mathrm{C}$. To identify macrophages and monocytes, cells were stained with antibodies against human CD14, CD36, CD68, and CD197. After staining, cells were washed and then resuspended in 1X DAPI in FACS buffer. Flow cytometry data were collected on an LSRFortessa or LSR II (BD Biosciences) and analyzed using FlowJo software (BD Biosciences).

\section{S RNA analysis}

Genomic DNA from oral microbiome samples from mice was isolated and prepared for 16S rRNA gene amplification (V1-V3 region) and sequencing at Majorbio (Shanghai, China) with a MiSeq 300 PE 
(Illumina MiSeq System) using primers (338F 5'-ACTCCTACGGGAGGCAGCAG-3'; 806R 5'GGACTACHVGGGTWTCTAAT-3'). The experiment was independently repeated four times. A total of four samples in each group were prepared $(n=4)$. Bioinformatics was performed with mothur and QIIME 2.0. Operational taxonomic unit (OTU) clustering was performed using USEARCH. Sequences were aligned and taxonomically assigned with the Silva database. To avoid biases due to different sequencing depths, OTU tables were rarefied to the lowest number of sequences per sample. Analyses were performed on the I-Sanger Cloud Platform (http://www.i-sanger.com). For alpha diversity, the Shannon index, Chao index, Simpson index, and Ace index at the OTU level were calculated. For beta diversity, PCA at the OTU level was performed, and analysis of similarities (ANOSIM) based on the Bray-Curtis distance was used to examine community differences. Average relative abundances of species are shown with Cicros plots and bar plots.

\section{Declarations}

\section{Data availability}

Raw and derived data supporting the work presented in this study are available from the corresponding author upon request. Source data are provided with this paper.

\section{Acknowledgements}

We are grateful to Chao-Jung Chu, Xiaoyu Wang, Chen Cui, and Wentao Jiang from West China Hospital of Stomatology, Sichuan University, Li Chen from Analytical and Test Center, Sichuan University, Xin Shi from Center of Stomatology, Tongji Hospital of Tongji Medical College, Huazhong University of Science and Technology, Jie Zhou and Huize Yan from Department of Biomedical Engineering, Columbia Univeristy, for expert technical assistance.

\section{Funding}

This work was supported by National Key R \& D Project (2018YFC1105701) and National Natural Science Foundation of China $(31870960,81901897)$ granted to S.Z., National Natural Science Foundation of China $(81970944,81991502)$ granted to L.Z., NIH AR073935 granted to K.W.L..

\section{Author contributions:}

H.H., W.P., and Y.W. contributed equally to this work. H.H., Y.W., Y.W., H.K., D.S., B.H., TC.H., YH.L., C.H.Q. and J.S. contributed to the collection of experimental data. H.H., Y.W., Y.W., H.K.,D.S. and TC.H. analyzed the data. H.H., W.P., Y.W., H.K., D.S., TC.H. and B.S. contributed to writing and revising the paper. S.Z., L.Z., and K.W.L. supervised the research.

\section{Competing interests:}

The authors declare that they have no competing interests. 


\section{*Corresponding authors:}

Shengmin Zhang, Advanced Biomaterials and Tissue Engineering Center and Department of Biomedical Engineering, Huazhong University of Science and Technology, Wuhan, Hubei 430074, China, E-mail: smzhang@mail.hust.edu.cn

Lei Zhao, State Key Laboratory of Oral Diseases and National Clinical Research Center for Oral Diseases, West China Hospital of Stomatology, Sichuan University, Chengdu, Sichuan 610041, China, E-mail: jollyzldoc@163.com

Kam W Leong, Department of Biomedical Engineering and Department of Systems Biology, Columbia University Medical Center, New York, NY 10032, USA, E-mail: kam.leong@columbia.edu

\section{References}

1 Crump, K. E. \& Sahingur, S. E. Microbial Nucleic Acid Sensing in Oral and Systemic Diseases. Journal of dental research 95, 17-25, doi:10.1177/0022034515609062 (2016).

2 Liwinski, T., Zheng, D. \& Elinav, E. The microbiome and cytosolic innate immune receptors. Immunol Rev, doi:10.1111/imr.12901 (2020).

3 Gu, Y. \& Han, X. Toll-Like Receptor Signaling and Immune Regulatory Lymphocytes in Periodontal Disease. Int J Mol Sci 21, doi:10.3390/ijms21093329 (2020).

4 Takeuchi, O. \& Akira, S. Pattern Recognition Receptors and Inflammation. Cell 140, 805-820, doi:https://doi.org/10.1016/j.cell.2010.01.022 (2010).

5 Paludan, S. R. \& Bowie, A. G. Immune sensing of DNA. Immunity 38, 870-880, doi:10.1016/j.immuni.2013.05.004 (2013).

6 Abuhussein, $\mathrm{H}$. et al. The role of factors associated with apoptosis in assessing periodontal disease status. Journal of periodontology 85, 1086-1095, doi:10.1902/jop.2013.130095 (2014).

7 Jahr, S. et al. DNA fragments in the blood plasma of cancer patients: quantitations and evidence for their origin from apoptotic and necrotic cells. Cancer research 61, 1659-1665 (2001).

8 Breitbach, S., Tug, S. \& Simon, P. Circulating cell-free DNA: an up-coming molecular marker in exercise physiology. Sports medicine (Auckland, N.Z.) 42, 565-586, doi:10.2165/11631380-00000000000000 (2012).

9 Sahingur, S. E. et al. DNA from Porphyromonas gingivalis and Tannerella forsythia induce cytokine production in human monocytic cell lines. Mol Oral Microbio/ 25, 123-135, doi:10.1111/j.20411014.2009.00551.x (2010). 
$10 \mathrm{Kim}, \mathrm{Y}$. et al. Toll-like receptor 9 mediates oral bacteria-induced IL-8 expression in gingival epithelial cells. Immunol Cell Bio/ 90, 655-663, doi:10.1038/icb.2011.85 (2012).

11 Sahingur, S. E., Xia, X. J. \& Schifferle, R. E. Oral bacterial DNA differ in their ability to induce inflammatory responses in human monocytic cell lines. Journal of periodontology $83,1069-1077$, doi:10.1902/jop.2011.110522 (2012).

12 Kim, P. D. et al. Toll-Like Receptor 9-Mediated Inflammation Triggers Alveolar Bone Loss in Experimental Murine Periodontitis. Infection and immunity 83, 2992-3002, doi:10.1128/IAl.00424-15 (2015).

13 Ziebolz, D. et al. Periodontal bacteria DNA findings in human cardiac tissue - Is there a link of periodontitis to heart valve disease? International journal of cardiology 251, 74-79, doi:10.1016/j.ijcard.2017.09.001 (2018).

14 Fardini, Y., Chung, P., Dumm, R., Joshi, N. \& Han, Y. W. Transmission of diverse oral bacteria to murine placenta: evidence for the oral microbiome as a potential source of intrauterine infection. Infection and immunity 78, 1789-1796, doi:10.1128/IAl.01395-09 (2010).

15 Rykova, E. et al. Circulating DNA in rheumatoid arthritis: pathological changes and association with clinically used serological markers. Arthritis Res Ther 19, 85-85, doi:10.1186/s13075-017-1295-z (2017).

16 Zhong, X. Y. et al. Increased concentrations of antibody-bound circulatory cell-free DNA in rheumatoid arthritis. Clinical chemistry 53, 1609-1614, doi:10.1373/clinchem.2006.084509 (2007).

17 Lee, J. et al. Nucleic acid-binding polymers as anti-inflammatory agents. Proc Natl Acad Sci US A 108, 14055-14060, doi:10.1073/pnas.1105777108 (2011).

18 Stearns, N. A., Lee, J., Leong, K. W., Sullenger, B. A. \& Pisetsky, D. S. The inhibition of anti-DNA binding to DNA by nucleic acid binding polymers. PLoS One 7, e40862, doi:10.1371/journal.pone.0040862 (2012).

19 Naqvi, I. et al. Polymer-Mediated Inhibition of Pro-invasive Nucleic Acid DAMPs and Microvesicles Limits Pancreatic Cancer Metastasis. Mol Ther 26, 1020-1031, doi:10.1016/j.ymthe.2018.02.018 (2018).

20 Holl, E. K. et al. Scavenging nucleic acid debris to combat autoimmunity and infectious disease. Proc Natl Acad Sci U S A 113, 9728-9733, doi:10.1073/pnas.1607011113 (2016).

21 Dawulieti, J. et al. Treatment of severe sepsis with nanoparticulate cell-free DNA scavengers. Sci $\operatorname{Adv}$ 6, eaay7148, doi:10.1126/sciadv.aay7148 (2020).

22 Peng, B. et al. Tuned Cationic Dendronized Polymer: Molecular Scavenger for Rheumatoid Arthritis Treatment. Angew Chem Int Ed Eng/ 58, 4254-4258, doi:10.1002/anie.201813362 (2019). 
23 Liang, H. et al. Cationic nanoparticle as an inhibitor of cell-free DNA-induced inflammation. Nat Commun 9, 4291, doi:10.1038/s41467-018-06603-5 (2018).

24 Wang, Y., Hao, H. \& Zhang, S. Lysozyme loading and release from Se doped hydroxyapatite nanoparticles. Materials science \& engineering. C, Materials for biological applications 61, 545-552, doi:10.1016/j.msec.2015.12.060 (2016).

25 Pajor, K., Pajchel, L., Kolodziejska, B. \& Kolmas, J. Selenium-Doped Hydroxyapatite NanocrystalsSynthesis, Physicochemical Properties and Biological Significance. Crystals 8, 188 (2018).

26 Apon, A. \& Kamble, P. Role of trace mineral in periodontal health: a review. Clinical Trials in Degenerative Diseases 4, 30, doi:10.4103/2542-3975.260960 (2019).

27 Gaur, S. \& Agnihotri, R. Trace Mineral Micronutrients and Chronic Periodontitis-a Review. Biological trace element research 176, 225-238, doi:10.1007/s12011-016-0832-y (2017).

28 Wang, Y. et al. Dual functional selenium-substituted hydroxyapatite. Interface focus 2, 378-386, doi:10.1098/rsfs.2012.0002 (2012).

29 Wang, Y. et al. In Vitro and in Vivo Mechanism of Bone Tumor Inhibition by Selenium-Doped Bone Mineral Nanoparticles. ACS nano 10, 9927-9937, doi:10.1021/acsnano.6b03835 (2016).

30 Marchesan, J. et al. An experimental murine model to study periodontitis. Nature Protocols 13, 2247-2267, doi:10.1038/s41596-018-0035-4 (2018).

31 Abe, T. \& Hajishengallis, G. Optimization of the ligature-induced periodontitis model in mice. J Immunol Methods 394, 49-54, doi:10.1016/j.jim.2013.05.002 (2013).

32 Pan, W., Wang, Q. \& Chen, Q. The cytokine network involved in the host immune response to periodontitis. International journal of oral science 11, 30, doi:10.1038/s41368-019-0064-z (2019).

33 Zhou, L. N. et al. Macrophage polarization in human gingival tissue in response to periodontal disease. Oral Dis 25, 265-273, doi:10.1111/odi.12983 (2019).

34 Zhuang, Z. et al. Induction of M2 Macrophages Prevents Bone Loss in Murine Periodontitis Models. Journal of dental research 98, 200-208, doi:10.1177/0022034518805984 (2019).

35 Stewart, D. A., Yang, Y., Makowski, L. \& Troester, M. A. Basal-like breast cancer cells induce phenotypic and genomic changes in macrophages. Mol Cancer Res 10, 727-738, doi:10.1158/15417786.MCR-11-0604 (2012).

36 Hajishengallis, G. Periodontitis: from microbial immune subversion to systemic inflammation. Nat Rev Immuno/ 15, 30-44, doi:10.1038/nri3785 (2015). 
37 Dwivedi, D. J. et al. Prognostic utility and characterization of cell-free DNA in patients with severe sepsis. Crit Care 16, R151, doi:10.1186/cc11466 (2012).

38 Dong, C. et al. Identification of Specific Joint-Inflammatogenic Cell-Free DNA Molecules From Synovial Fluids of Patients With Rheumatoid Arthritis. Frontiers in immunology 11, 662, doi:10.3389/fimmu.2020.00662 (2020).

39 Heitzer, E., Auinger, L. \& Speicher, M. R. Cell-Free DNA and Apoptosis: How Dead Cells Inform About the Living. Trends Mol Med 26, 519-528, doi:10.1016/j.molmed.2020.01.012 (2020).

40 Preissner, K. T. \& Herwald, H. Extracellular nucleic acids in immunity and cardiovascular responses: between alert and disease. Thrombosis and haemostasis 117, 1272-1282, doi:10.1160/th-1611-0858 (2017).

41 Whanger, P. D. Selenium and its relationship to cancer: an update. The British journal of nutrition 91, 11-28, doi:10.1079/bjn20031015 (2004).

42 Hoeg, A. et al. Bone turnover and bone mineral density are independently related to selenium status in healthy euthyroid postmenopausal women. The Journal of clinical endocrinology and metabolism 97, 4061-4070, doi:10.1210/jc.2012-2121 (2012).

43 Negi, S., Das, D. K., Pahari, S., Nadeem, S. \& Agrewala, J. N. Potential Role of Gut Microbiota in Induction and Regulation of Innate Immune Memory. Frontiers in immunology 10, 2441, doi:10.3389/fimmu.2019.02441 (2019).

44 Anderton, H., Wicks, I. P. \& Silke, J. Cell death in chronic inflammation: breaking the cycle to treat rheumatic disease. Nat Rev Rheumatol, doi:10.1038/s41584-020-0455-8 (2020).

45 Faas, M. M. \& de Vos, P. Mitochondrial function in immune cells in health and disease. Biochimica et biophysica acta. Molecular basis of disease 1866, 165845, doi:10.1016/j.bbadis.2020.165845 (2020).

46 Gambardella, S. et al. ccf-mtDNA as a Potential Link Between the Brain and Immune System in Neuro-Immunological Disorders. Frontiers in immunology 10, 1064, doi:10.3389/fimmu.2019.01064 (2019).

47 Curtis, M. A., Diaz, P. I. \& Van Dyke, T. E. The role of the microbiota in periodontal disease. Periodontology 2000 83, 14-25, doi:10.1111/prd.12296 (2020).

48 Wilensky, A. et al. Dendritic cells and their role in periodontal disease. Oral Dis 20, 119-126, doi:10.1111/odi.12122 (2014).

49 Yang, J. et al. Enhanced activity of macrophage M1/M2 phenotypes in periodontitis. Arch Oral Biol 96, 234-242, doi:10.1016/j.archoralbio.2017.03.006 (2018). 
50 Tonetti, M. S., Greenwell, H. \& Kornman, K. S. Staging and grading of periodontitis: Framework and proposal of a new classification and case definition. Journal of periodontology 89 Suppl 1, S159-s172, doi:10.1002/jper.18-0006 (2018).

51 Abe, T. \& Hajishengallis, G. Optimization of the ligature-induced periodontitis model in mice. $J$ Immunol Methods 394, 49-54, doi:10.1016/j.jim.2013.05.002 (2013).

52 Abusleme, L. et al. Oral Microbiome Characterization in Murine Models. Bio-protocol 7, doi:10.21769/BioProtoc.2655 (2017).

\section{Figures}

A
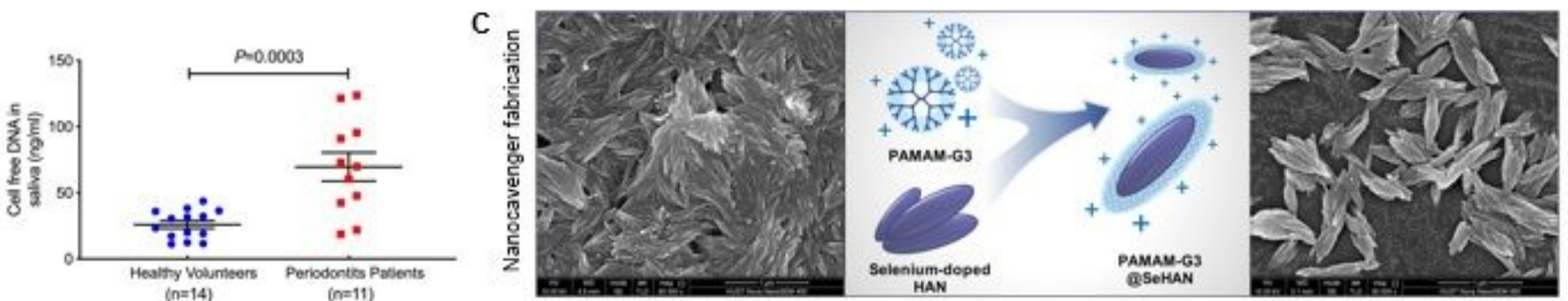

B
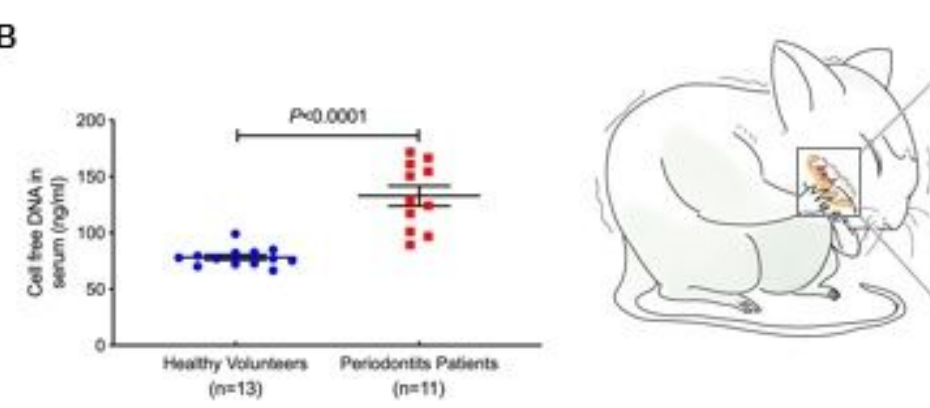

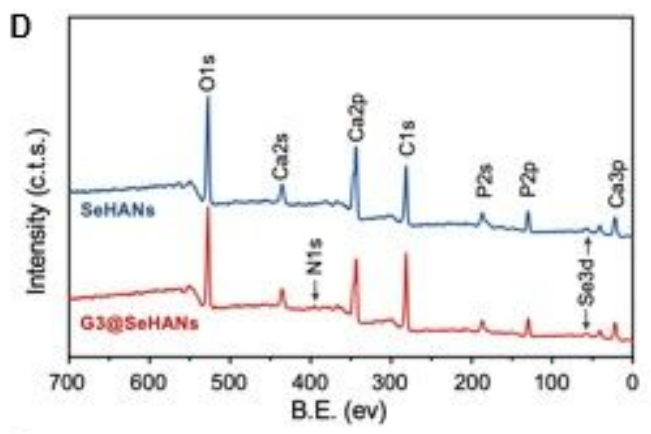

G
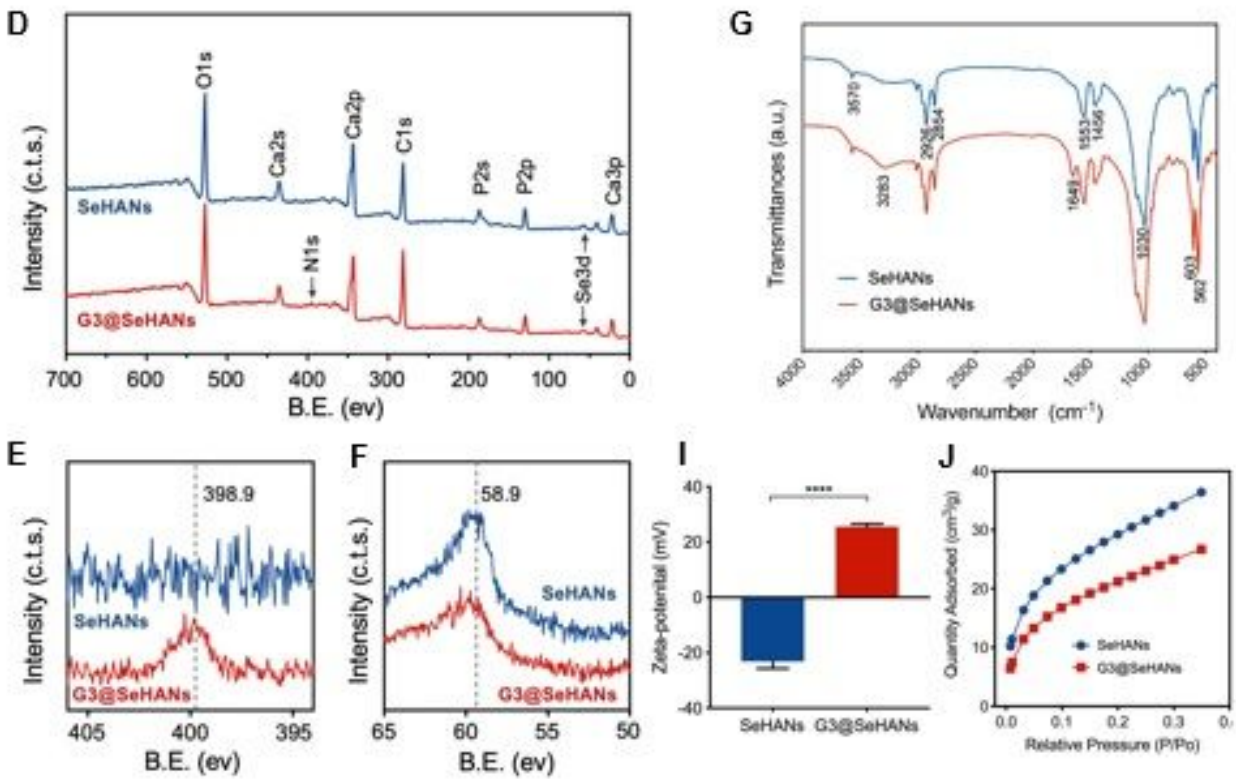

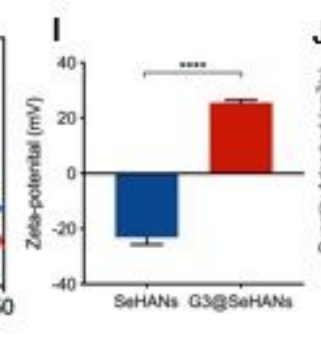

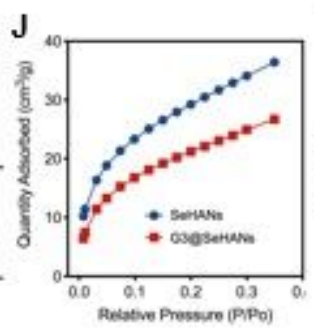

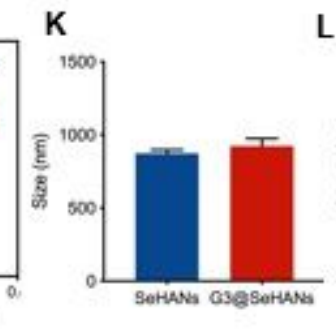

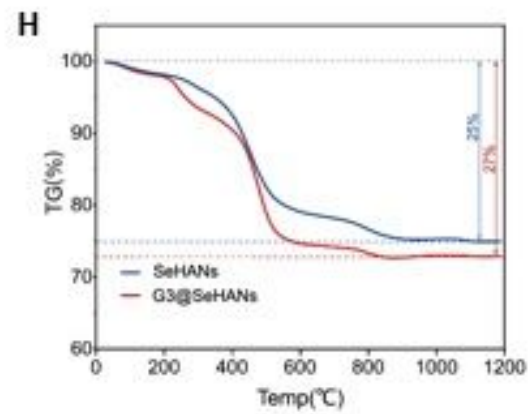

L

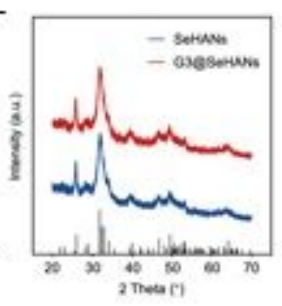

Figure 1 
Elevated cfDNA in saliva and serum of periodontitis patients, and characterization of cfDNA-binding nanoparticles. (A-B) cfDNA levels in (A) saliva of healthy volunteers $(n=14)$ and periodontitis patients $(n=11)$, and $(B)$ serum of healthy volunteers $(n=13)$ and periodontitis patients $(n=11)$. Data are means \pm SEM; differences were assessed by Student's t-test. (C) Top: SEM imaging of SeHANs before and after coating with PAMAM-G3. Bottom: Schematic of mechanism by which cfDNA promotes bone loss; cfDNAscavenging nanoparticles may prevent bone loss and alleviate periodontitis. (D-F) XPS spectra. The N1s photoelectron peak (398.9 eV) is present only in G3@SeHANs; Se3d peaks (58.9 eV) are present in both SeHANs and G3@SeHANs. (G) FTIR spectra. Both SeHANs and G3@SeHANs exhibit characteristic phosphate bands at 562,603 , and $1030 \mathrm{~cm}-1$, and bands corresponding to $0-S e-0$ bending vibration (783 $\mathrm{cm}-1)$ and hydroxyl groups (3570 cm-1). Peaks due to amino stretching vibration (3283 cm-1) and bending vibration of the amide N-H bond (1649 cm-1) are present only in G3@SeHANs. (H) The thermal decomposition rate of the nanoparticles changes after coating with PAMAM-G3. (I) Zeta potential of SeHANs and G3@SeHANs. Data are means \pm SEM; differences were assessed by Student's t-test $(n=5)$. (J) BET analysis of SeHANs and G3@SeHANs. (K) Size measurement of SeHANs and G3@SeHANs. Data are means \pm SEM; differences were assessed by Student's t-test. (L) XRD analysis of SeHANs and G3@SeHANs. ${ }^{*}<<0.05,{ }^{* *} P<0.01,{ }^{*} * * P<0.001, * * \star * P<0.0001$.
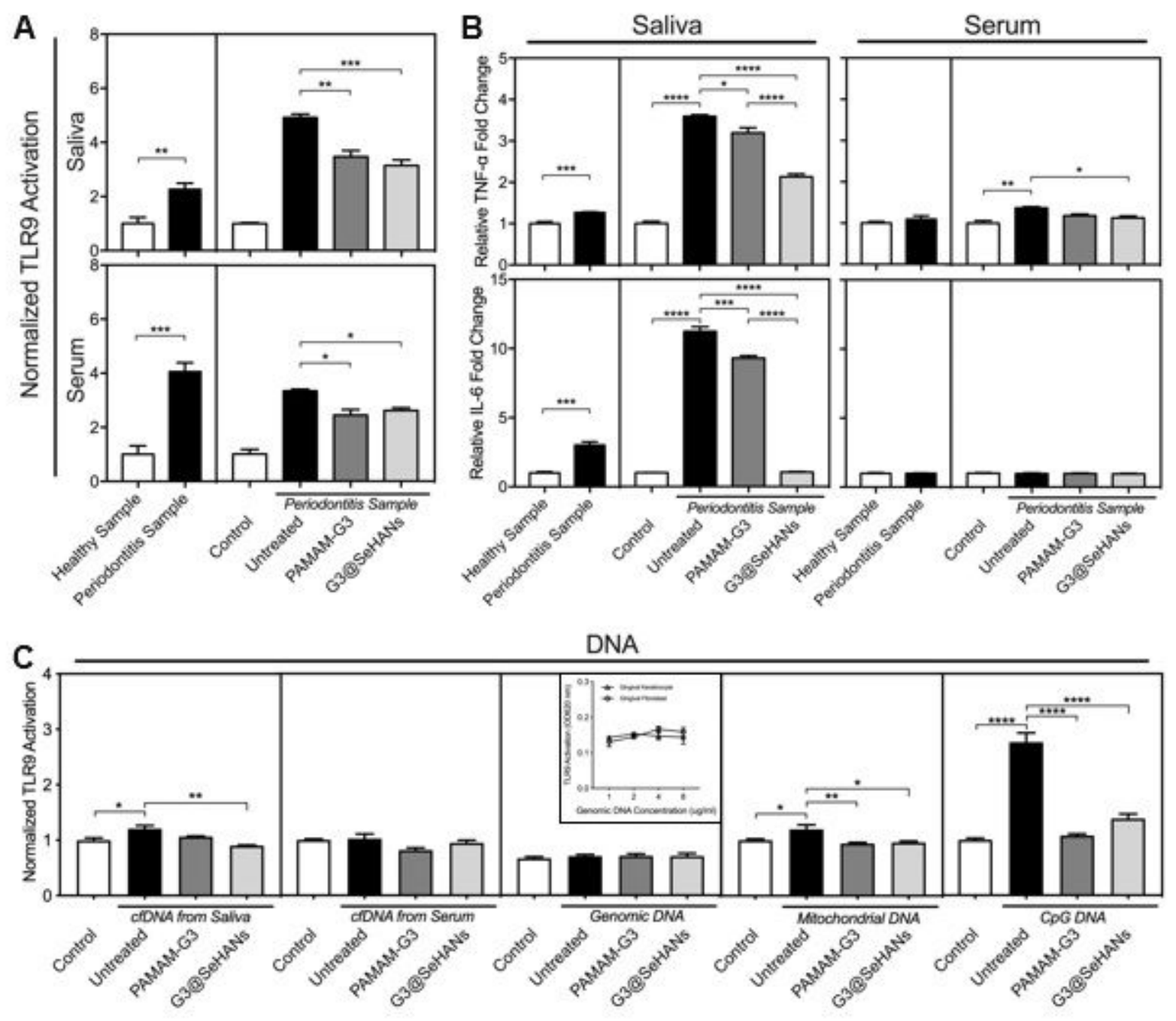
Figure 2

G3@SeHANs block periodontitis-related cellular inflammation in vitro by scavenging cfDNA. (A) Activation of HEK-TLR9 reporter cells, and (B) TNF-a and IL-6 expression by RAW 264.7 macrophages induced by healthy human saliva, periodontitis patient saliva, healthy human serum, or periodontitis patient serum in the absence or presence of PAMAM-G3 $(2 \mu \mathrm{g} / \mathrm{mL})$ or G3@SeHANs $(10 \mu \mathrm{g} / \mathrm{mL})$ for $24 \mathrm{~h}$. (C) Activation of HEK-TLR9 reporter cells by cfDNA from periodontitis patient saliva or serum, genomic DNA, mtDNA, and CpG DNA in the absence or presence of PAMAM-G3 $(2 \mu \mathrm{g} / \mathrm{mL})$ or G3@SeHANs (10 $\mu \mathrm{g} / \mathrm{mL}$ ) for $24 \mathrm{~h}$. Data are means $\pm \mathrm{SEM} ; \mathrm{n}=3$ independent experiments; ${ }^{*} \mathrm{P}<0.05,{ }^{\star \star} P<0.01,{ }^{\star * *} \mathrm{P}<0.001$, $* * * * P<0.0001$ by Student's t-test and one-way ANOVA with Tukey's multiple comparison test.

A

- Ligature - Saliva collection - Blood collection $*$ Micro CT

* Sacrifice $*$ Tissue collection $\square$ Histology

$\boldsymbol{\nabla}$ Local injection with materials $\nabla$ Materials smear on the gum around the ligature

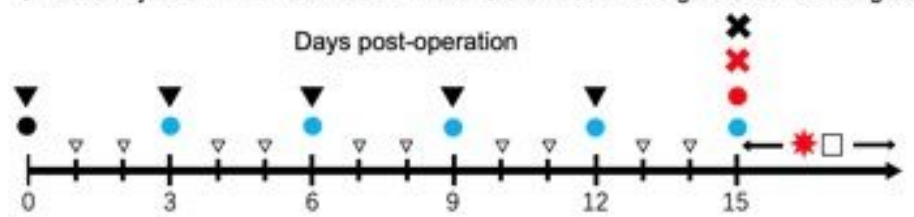

Group 1: Normat; 2: Ligature + PBS: 3: Ligature + PAMAM-G3; 4: Ligature + G3@SeHANs

$\mathrm{C}$
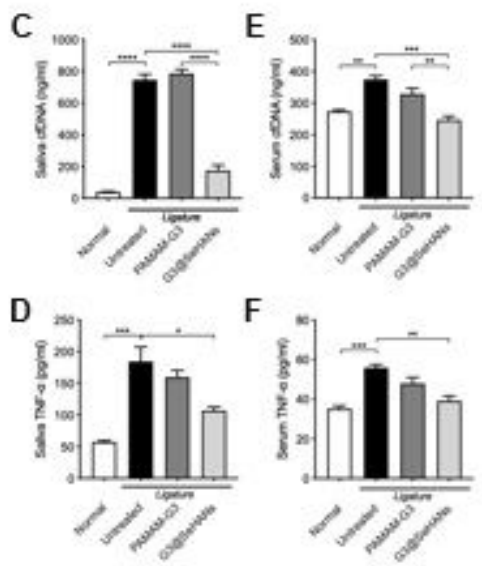

G
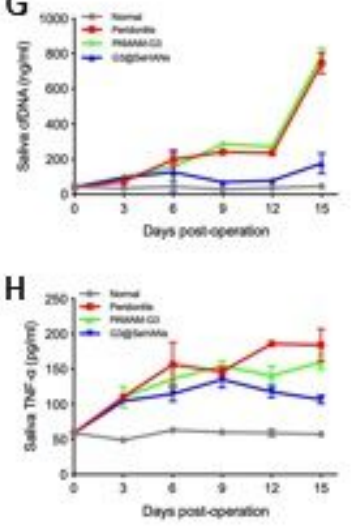

B

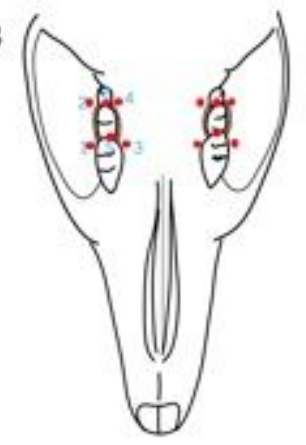

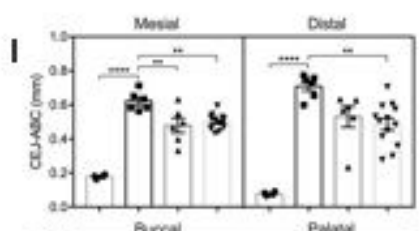
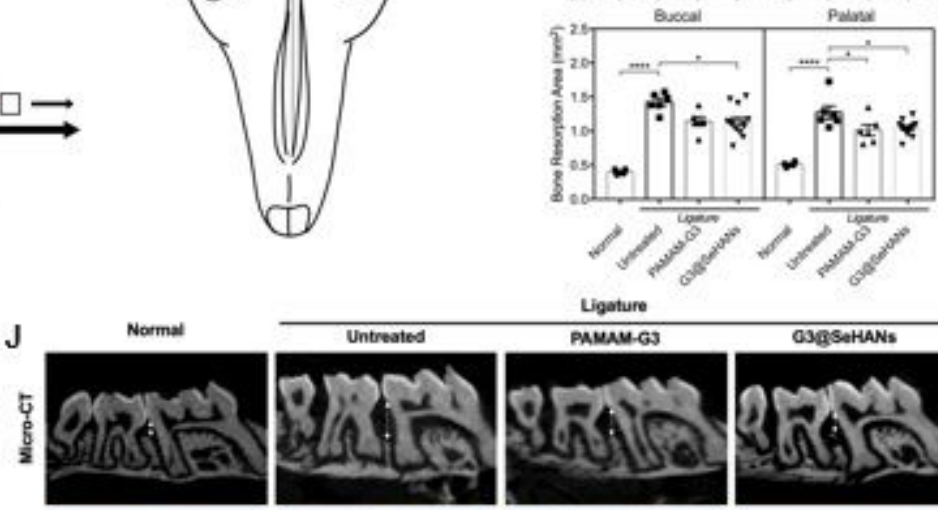

Ligature
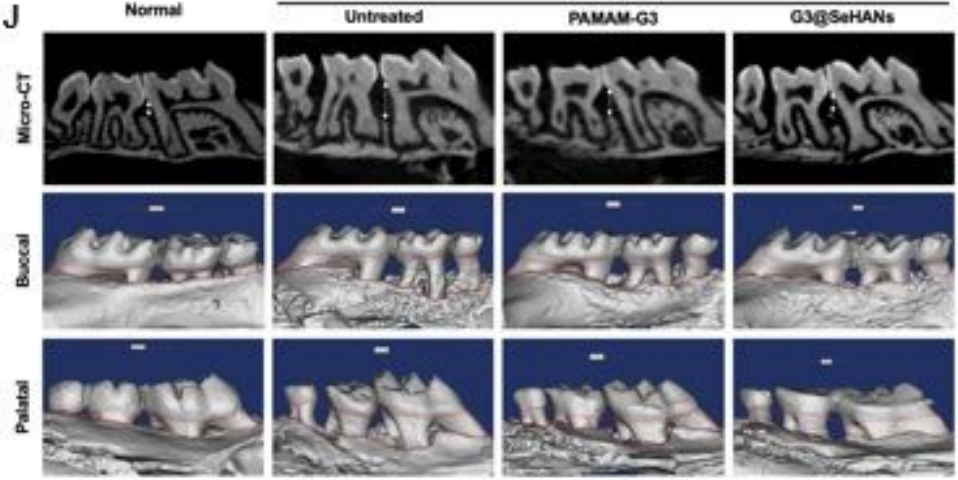

\section{Figure 3}

G3@SeHANs alleviate inflammatory bone loss in ligature-induced periodontitis. (A-B) Experimental schedule of in vivo study. PAMAM-G3 $(200 \mu \mathrm{g} / \mathrm{mL})$, G3@SeHANs $(1 \mathrm{mg} / \mathrm{mL})$, or PBS (control group) were administered locally by injection with a microsyringe into gingival tissue at $5 \mu \mathrm{L}$ per site at 6 sites around the ligature: the mesiobuccal gingiva, distobuccal gingiva, mesiopalatal gingiva, distopalatal gingiva, and mesial and distal gingival papilla. Microinjections were performed on days $0,3,6,9$, and 12 . On experimental days without injections, materials or PBS were noninvasively smeared on the gingiva with disposable microapplicators. Both sides of the mice were treated with the same materials. Levels of (C) saliva cfDNA, (D) saliva TNF-a, (E) serum cfDNA, and (F) serum TNF-a at 15 days post-operation. Data are means \pm SEM; $n=3$ samples per group; ${ }^{*} P<0.05$, ${ }^{*} P<0.01,{ }^{*} * * P<0.001,{ }^{*} * \star * P<0.0001$ by one-way ANOVA with Tukey's multiple comparison test. (G-H) Changes in cfDNA and TNF-a concentrations in the 
saliva of mice. Data are means $\pm \mathrm{SEM} ; \mathrm{n}=3$ samples per group. (I) Bone loss measured by the vertical distance between the cementoenamel junction (CEJ) and alveolar bone crest (ABC) (CEJ-ABC) and the bone resorption area. Data are means $\pm S E M ; * P<0.05,{ }^{\star} * P<0.01$, ${ }^{\star * \star *} P<0.0001$ by one-way ANOVA with Tukey's multiple comparison test. $(\mathrm{J})$ Micro-CT scanning and 3D reconstruction of the bone loss.

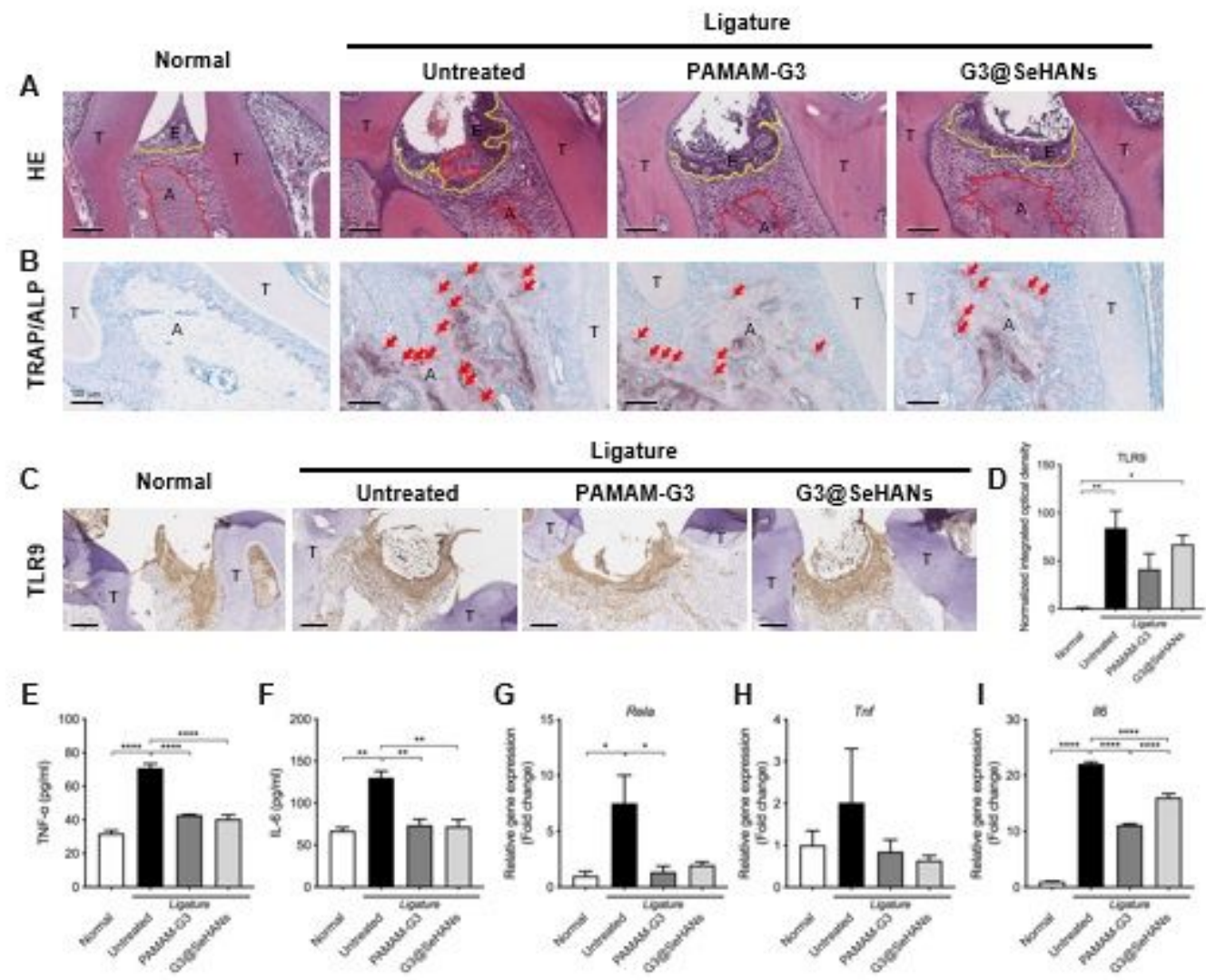

\section{Figure 4}

G3@SeHANs exhibit better bone protection and inhibition of cellular proinflammatory response than soluble PAMAM-G3. (A) H\&E staining of periodontal tissues on day 15 after G3@SeHAN administration. Scale bars, $100 \mu \mathrm{m}$. Inflammatory cell infiltration into the epithelium and bone destruction were evident in the untreated model, whereas G3@SeHAN treatment prevented these pathological changes. $(E$, epithelium; A, alveolar bone; T, tooth; yellow line indicates epithelial tissue). (B) TRAP/ALP staining of periodontal tissues on day 15 after G3@SeHAN administration. Scale bars, $100 \mu \mathrm{m}$. The number of osteoclasts (red arrows) in the untreated group was higher than in the DNA scavenger-treated groups. (C) IHC staining of TLR9 in periodontal tissues on day 15 after G3@SeHAN administration. Scale bars, 100 $\mu \mathrm{m}$. TLR9 expression increased significantly in the untreated group; treatment with G3@SeHAN or PAMAM-G3 reduced TLR9 expression. (D) Expression of TLR9 in the epithelium of periodontal tissues. (EF) TNF- $a$ and IL-6 levels in periodontal tissues. (G-I) Relative gene expression of Rela, Tnf, and II6 in periodontal tissues. Data are means $\pm S E M ; n=3$ samples per group; ${ }^{*} P<0.05,{ }^{*} P<0.01,{ }^{\star} * \star P<0.001$, $\star \star \star \star P<0.0001$ by one-way ANOVA with Tukey's multiple comparison test. 
A

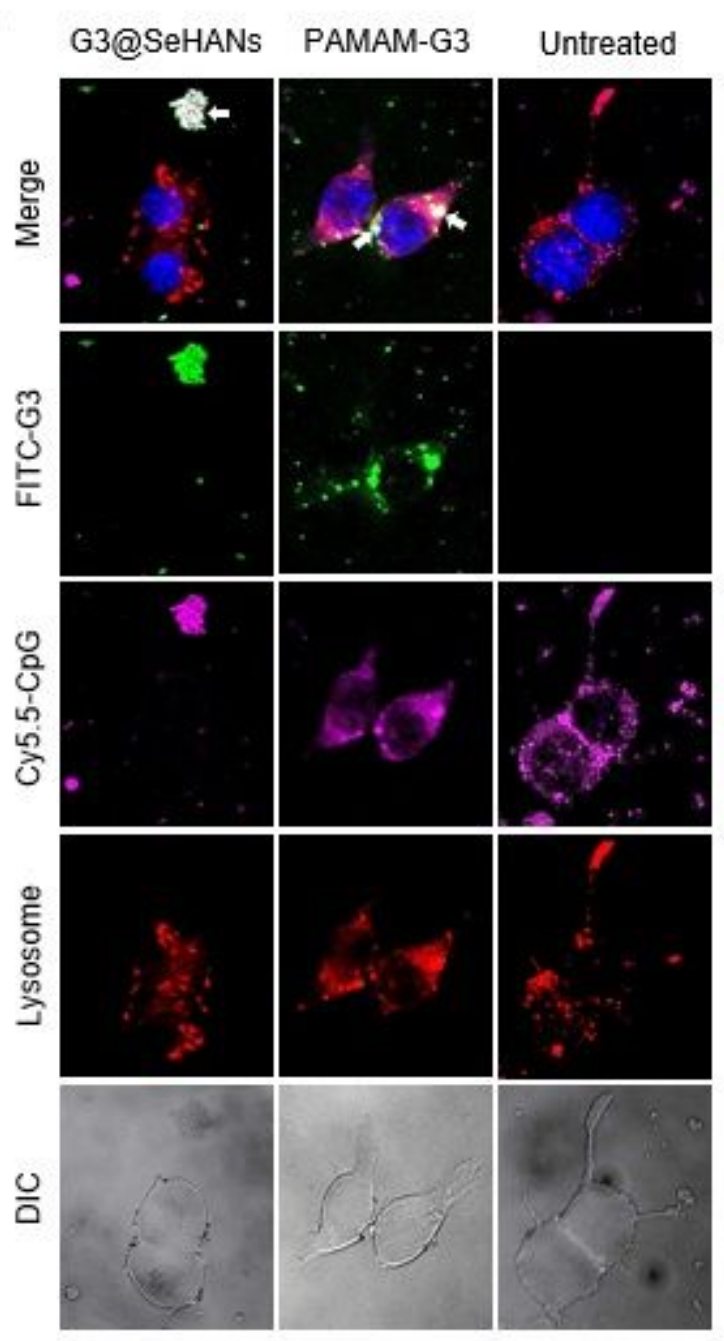

B

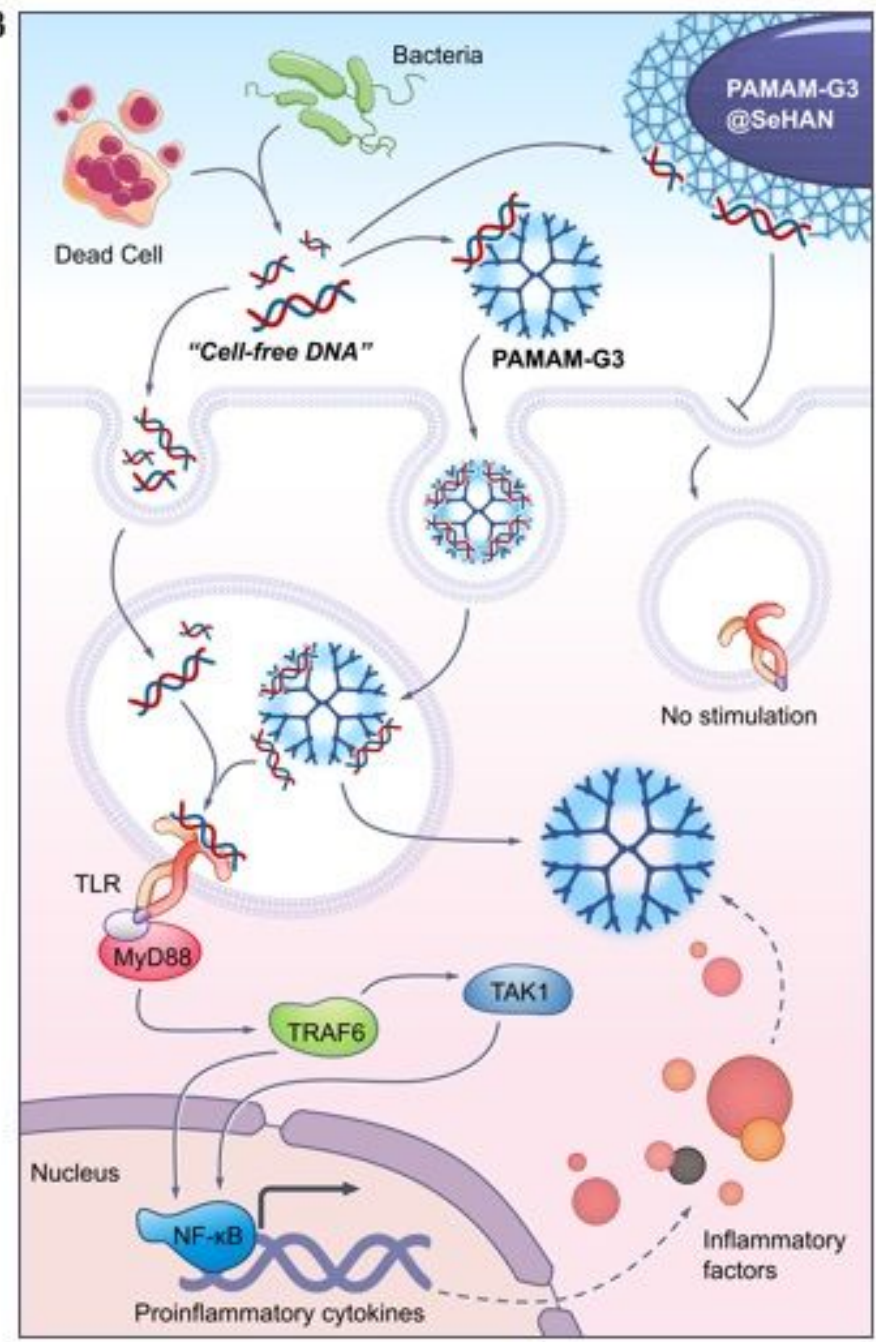

\section{Figure 5}

Scavenging of cfDNA by G3@SeHANs and PAMAM-G3. (A) Enlarged images show intracellular localization of $\mathrm{CpG}$ oligonucletides and cationic materials in RAW 264.7 cells after a $12 \mathrm{~h}$ incubation. Colocalized CpG and cationic nanoparticles appear as white spots, and are indicated by arrows. (B) Scavenging mechanisms of G3@SeHANs and PAMAM-G3. 

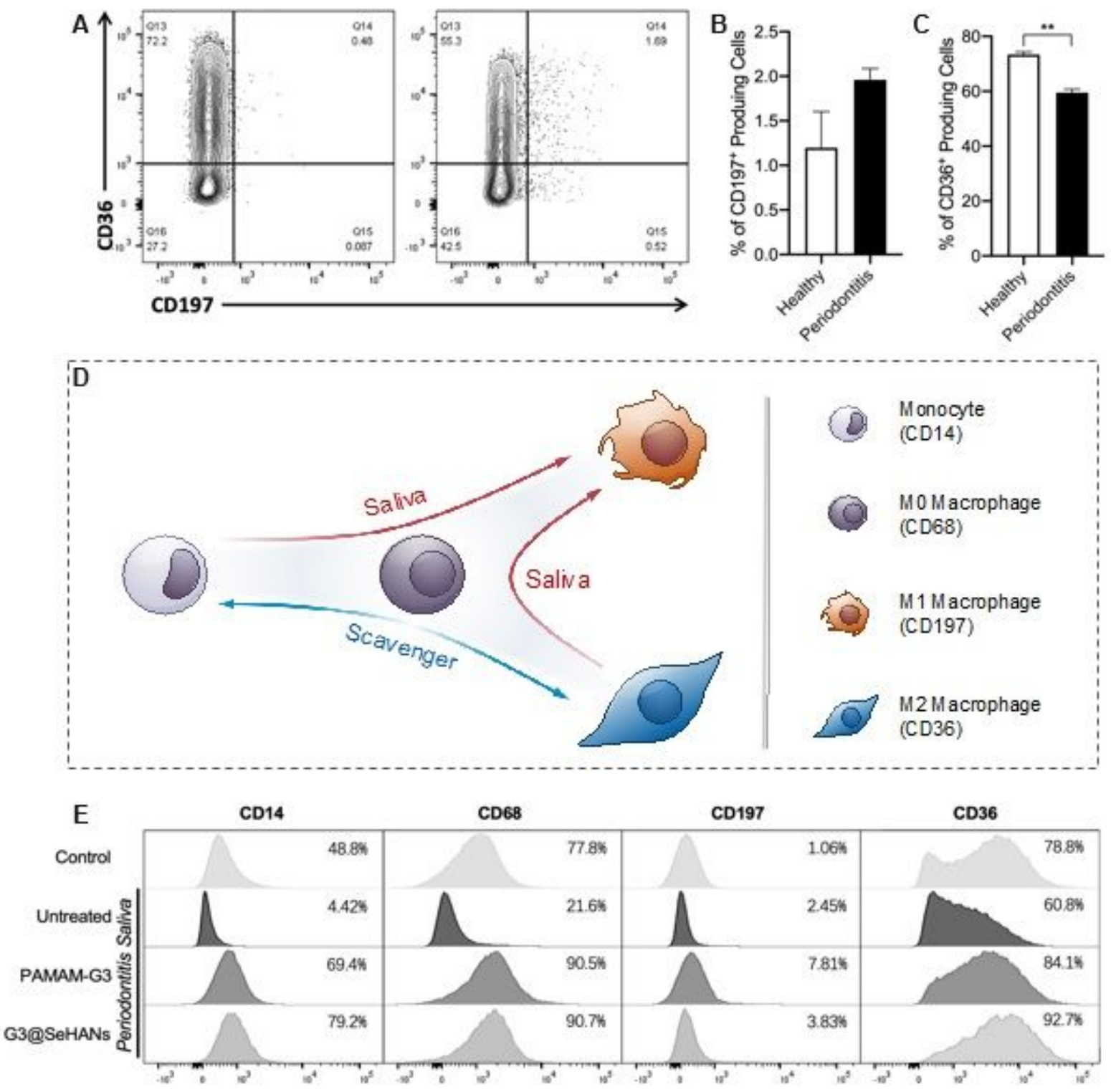

Figure 6

Effects of cationic scavengers on macrophage polarization. (A-C) Expression of the M1 marker CD197 and the M2 marker CD36 in THP-1 cells activated by periodontitis saliva. Data are means \pm SEM; differences were assessed by Student's t-test ( $n=3$ samples per group). (D) Schematic of macrophage polarization by periodontitis saliva and role of cationic cfDNA scavengers. (E) Effects of periodontitis saliva and cationic scavengers on expression of CD14, CD 68, CD 197, and CD36 in THP-1 cells. ${ }^{*} \mathrm{P}<0.05$, $\star * P<0.01$.

\section{Supplementary Files}

This is a list of supplementary files associated with this preprint. Click to download. 
- NCSupplementaryMaterials.pdf

Page 30/30 